\title{
Towards a Nonperturbative Theory of Hydrodynamic Turbulence: Fusion Rules, Exact Bridge Relations and Anomalous Viscous Scaling Functions
}

\author{
Victor L'vov甘 and Itamar Procaccia \\ ${ }^{* \dagger}$ Department of Chemical Physics, The Weizmann Institute of Science, Rehovot 76100, Israel, \\ ${ }^{*}$ Institute of Automation and Electrometry, Ac. Sci. of Russia, 630090, Novosibirsk, Russia
}

\begin{abstract}
of equations is decoupled in the sense that the correlations of In this paper we address nonperturbative aspects of the analytic theory of hydrodynamic turbulence. Of paramount importance for this theory are the "fusion rules" that describe the asymptotic properties of $n$-point correlation functions when some of the coordinates tend toward one other. We first derive here, on the basis of the Navier-Stokes equations, a set of fusion rules for correlations of velocity differences when all the separation are in the inertial interval. Using this set of fusion rules we consider the standard hierarchy of equations relating the $n$-th order correlations (originating from the viscous term in the Navier-Stokes equations) to $n+1$ 'th order (originating from the nonlinear term) and demonstrate that for fully unfused correlations the viscous term is negligible. Consequently the hierarchic chain of equations is decoupled in the sense that the correlations of $n+1$ 'th order satisfy a homogeneous equation that may exhibit anomalous scaling solutions. Using the same hierarchy of equations when some separations go to zero we derive a second set of fusion rules for correlations with differences in the viscous range. The latter includes gradient fields. We demonstrate that every n'th order correlation function of velocity differences $\mathcal{F}_{n}\left(R_{1}, R_{2}, \ldots\right)$ exhibits its own cross-over length $\eta_{n}$ to dissipative behavior as a function of, say, $R_{1}$. This length depends on $n$ and on the remaining separations $R_{2}, R_{3}, \ldots$. When all these separations are of the same order $R$ this length scales like $\eta_{n}(R) \sim \eta(R / L)^{x_{n}}$ with $x_{n}=\left(\zeta_{n}-\zeta_{n+1}+\zeta_{3}-\zeta_{2}\right) /\left(2-\zeta_{2}\right)$, with $\zeta_{n}$ being the scaling exponent of the $n$ 'th order structure function. We derive a class of exact scaling relations bridging the exponents of correlations of gradient fields to the exponents $\zeta_{n}$ of the $n$ 'th order structure functions. One of these relations is the well known "bridge relation" for the scaling exponent of dissipation fluctuations $\mu=2-\zeta_{6}$.
\end{abstract}

\section{INTRODUCTION}

In a recent series of papers [1,2, 3, 3 , 1 we developed an approach to the theory of the universal scaling properties that characterize the statistical invariants of fully developed turbulent flows. The main technical tool of this approach was renormalized perturbation theory which allows, after exact resummations, to obtain insights about the non-perturbative properties of the universal statistics of turbulence. The main nonperturbative results which were obtained are (i) Hydrodynamic interactions are local in physical-space and in scale-space once the sweeping effects are removed. (ii) The inner, viscous scale which appears perturbatively as an Ultra-Violet cutoff leads non-perturbatively to the anomalous scaling of correlations involving velocity gradients. (iii) The outer scale of turbulence appears in the theory as a renormalization scale after infinite resummations of a renormalized perturbation series that converges order by order. The aim of this paper is to present new nonperturbative results that together with the previous findings begin to assemble to a solid structure of the theory of hydrodynamic turbulence. Brief presentations of some of the new results were offered in [5, 6, 7, 8].

The issue under study is not new; decades of experimental and theoretical attention (see for example [9, 10, 12, 13, 14, ) have been devoted to two types of simultaneous correlation functions; the first type includes the structure functions of velocity differences,

$$
\begin{aligned}
S_{n}(\boldsymbol{R}) & =\left\langle\left|\boldsymbol{w}\left(\boldsymbol{r}, \boldsymbol{r}^{\prime}\right)\right|^{n}\right\rangle, \\
\boldsymbol{w}\left(\boldsymbol{r}, \boldsymbol{r}^{\prime}\right) & \equiv \boldsymbol{u}\left(\boldsymbol{r}^{\prime}\right)-\boldsymbol{u}(\boldsymbol{r}), \quad \boldsymbol{R} \equiv \boldsymbol{r}^{\prime}-\boldsymbol{r},
\end{aligned}
$$

where $\langle\ldots\rangle$ stands for a suitably defined ensemble average. A second type of correlations include gradients of the velocity field. An important example is the rate $\epsilon(\mathbf{r}, t)$ at which energy is dissipated into heat due to viscous damping. This rate is roughly $\nu|\nabla \boldsymbol{u}(\boldsymbol{r}, t)|^{2}$. An oftenstudied simultaneous correlation function of $\hat{\epsilon}(\boldsymbol{r}, t)=$ $\epsilon(\boldsymbol{r}, t)-\bar{\epsilon}$ is

$$
K_{\epsilon \epsilon}(\boldsymbol{R})=\langle\hat{\epsilon}(\boldsymbol{r}+\boldsymbol{R}) \hat{\epsilon}(\boldsymbol{r})\rangle .
$$

Within the famous Kolmogorov 1941 (K41) approach [15] to turbulence one predicts that for $R$ in the inertial range, i.e $\eta \ll R \ll L, S_{n}(R)$ is $(\bar{\epsilon} R)^{n / 3}$ and
*Electronic address: fnLvov@wis.weizmann.ac.il

${ }^{\dagger}$ Electronic address: cfProcac@weizmann.weizmann.ac.il,
URL: http://lvov.weizmann.ac.il

URL: http://chemphys.weizmann.ac.il/procaccia.html 
$K_{\epsilon \epsilon}(R) \simeq \nu^{2} \bar{\epsilon}^{4 / 3} R^{-8 / 3}$. Here $L$ and $\eta$ are respectively the outer scale of turbulence and the Kolmogorov viscous cutoff. $\bar{\epsilon}$ is the mean energy flux per unit time per unit mass.

Experimental measurements show that in some aspects Kolmogorov was remarkably close to the truth. The major aspect of his predictions, that the statistical quantities depend on the length scale $R$ as power laws, is corroborated by experiments. On the other hand, the predicted exponents seem not to be exactly realized. For example, the correlation $K_{\epsilon \epsilon}(R)$ decays according to a power law,

$$
K_{\epsilon \epsilon}(R) \propto R^{-\mu},
$$

for $\eta \ll R \ll L$, with $\mu$ having a numerical value of $0.2-0.3$ instead of $8 / 3$ [12]. The structure functions also behave as power laws,

$$
S_{n}(R) \propto R^{\zeta_{n}}
$$

but the numerical values of $\zeta_{n}$ deviate progressively from $n / 3$ when $n$ increases 10,13. Something fundamental seems to be missing.

The first positive answer within our approach appeared in the context of correlation functions of gradient fields rather than of the velocity differences [3 [16]. As indicated above the discrepancy in the exponent $\mu$ between the experimental value and the naive expectation is large. This gave hope that the explanation must lie close to the surface. Indeed, considering the perturbative scheme for $K_{\epsilon \epsilon}(R)$ leads immediately to the discovery of logarithmic ultraviolet divergences with some ultraviolet viscous scale $\tilde{\eta}$ acting as the renormalization scale. The summation of this infinite series results in a factor $(R / \tilde{\eta})^{2 \Delta}$ with some anomalous exponent $\Delta$ which is, generally speaking, of the order of unity. Additional calculations lead to the exact result $\Delta=2-\zeta_{2}$ and to the understanding that this result means that the renormalized perturbation series for the structure functions diverges in the limit $L \rightarrow \infty$ like $(L / R)^{\delta_{n}}$. The anomalous exponents $\delta_{n}$ are the deviations of the exponents of $S_{n}(R)$ from their K41 value. This is a very delicate and important point. It is not simple to see why a series each of whose terms converges when $L \rightarrow \infty$ still diverges as a whole. One can understand this as in re-summing the series of a desired function one finds an inhomogeneous equation whose inhomogeneous solutions are indeed $L$-independent. However, the equation possesses also homogeneous solutions which are inherently nonperturbative in nature and may have anomalous scaling. Homogeneous solutions must be matched with the boundary conditions, and this is the way that the outer scale appears in the theory. An important remaining step in the theoretical development is to understand how to compute the anomalous exponents $\delta_{n}$.

The divergence of the entire perturbation series for $S_{n}(R)$ with $L \rightarrow \infty$ forces us to seek a nonperturbative handle on the theory. One fact that may potentially lead to a nonperturbative control is the existence of a global balance between energy input and dissipation. This may be turned into a nonperturbative constraint on each $n$ th order structure function [4]. Using the Navier-Stokes equations one derives the set of equations of motion

$$
\frac{\partial S_{n}(R, t)}{\partial t}+D_{n}(R, t)=J_{n}(R, t),
$$

where $D_{n}$ and $J_{n}$ stem from the nonlinear and the viscous terms in the Navier Stokes equations respectively. In the stationary state the time derivative vanishes and one has the balance equation

$$
D_{n}(R)=J_{n}(R) .
$$

The evaluation of $D_{n}(R)$ does not pose conceptual difficulties. It was shown [ 1 t that it is of the order of $d S_{n+1} / d R$. The evaluation of $J_{n}(R)$ poses some delicate issues, but it was shown that it is proportional to $S_{n}(R) / S_{2}(R)$. This result is of course the famous hierarchic chain of equations of which many closure attempts have failed to provide the desired anomalous scaling solutions.

At the heart of this paper we offer a way out of this conundrum. The problem with Eq.(1.7) is that it refers to a "fully fused" quantity, and this fact leads to difficulties. What is meant by this is that the primitive object of the statistical theory of turbulence is not the structure function $S_{n}(R)$, but rather the "fully unfused" $n$-rank tensor correlation function of velocity differences:

$$
\begin{aligned}
& \boldsymbol{F}_{n}\left(\boldsymbol{r}_{1}, \boldsymbol{r}_{1}^{\prime} ; \boldsymbol{r}_{2}, \boldsymbol{r}_{2}^{\prime} ; \ldots ; \boldsymbol{r}_{n}, \boldsymbol{r}_{n}^{\prime}\right) \\
= & \left\langle\boldsymbol{w}\left(\boldsymbol{r}_{1}, \boldsymbol{r}_{1}^{\prime}\right) \boldsymbol{w}\left(\boldsymbol{r}_{2}, \boldsymbol{r}_{2}^{\prime}\right) \ldots \boldsymbol{w}\left(\boldsymbol{r}_{n}, \boldsymbol{r}_{n}^{\prime}\right)\right\rangle .
\end{aligned}
$$

In this quantity all the coordinates are distinct. The structure function $S_{n}(R)$ is obtained by fusing all the coordinates $\boldsymbol{r}_{i}$ into one point $\boldsymbol{r}$, and all the coordinates $\boldsymbol{r}_{i}^{\prime}$ into another point $\boldsymbol{r}+\boldsymbol{R}$. In this process of fusion one crosses the dissipative scale. To control this process we need to formulate the so-called "fusion rules" for turbulence, which govern the properties of $n$-point correlation functions when two or more coordinates "fuse" together. One cannot fuse blindly.

The main fundamental results of this paper are as follows:

1. Fusion rules in the inertial interval. The explanation of how fusion works was briefly given in [⿹] We begin this paper in Sections II and III with a detailed derivation of the fusion rules, considering all the possible relative geometries of the sets of fused and unfused points.

2. The homogeneous equation for the $(n+1)$-order correlation functions: the fusion rules allow us to consider in Section IV and V the generalized balance equation (4.7) for the fully unfused correlation functions. We demonstrate that for these quantities the hierarchic chain of equations decouples, 
since now the dissipative term vanishes when the viscosity $\nu$ goes to zero. In the case of Eq.(1.7) the term $J_{n}$ contains a correlation function with many fused coordinates. As a result (and cf. Section VI) it remains finite in the limit $\nu \rightarrow 0$. In the present case of fully unfused quantities we get in the limit $\nu \rightarrow 0$ a homogeneous equation involving only correlations of order $n+1$. The full analysis of this equation is not easy, and it deserves a considerable amount of attention in the future.

3. The existence of "viscous scaling functions". Having obtained the generalized balance equation we can now study precisely when, as a function of one separation distance (say $\left|\boldsymbol{r}_{1}^{\prime}-\boldsymbol{r}_{1}\right|$ ), the fully unfused correlation function crosses over to dissipative behavior. It turns out that the scale at which this happens depends on the rank $n$ and on the remaining inertial range distances. The dependence on the inertial range distances is characterized by anomalous exponents, and we compute them exactly in terms of the anomalous exponents $\zeta_{n}$. This is done in Section VI.

4. Exact bridge relations. With a precise evaluation of the viscous scaling functions and the fusion rules in hand we can consider correlation functions of gradient fields, and relate their scaling exponents to the anomalous exponents $\zeta_{n}$. The result of this exercise, which is presented in Section VII, is an infinite set of bridge relations. We consider correlations of the type

$$
\begin{aligned}
\mathcal{K}_{p \epsilon}^{(n)} \equiv & \left\langle\epsilon\left(\boldsymbol{x}_{1}\right) \epsilon\left(\boldsymbol{x}_{2}\right) \ldots \epsilon\left(\boldsymbol{x}_{p}\right) \boldsymbol{w}\left(\boldsymbol{r}_{1}, \boldsymbol{r}_{1}^{\prime}\right) \ldots \boldsymbol{w}\left(\boldsymbol{r}_{n}, \boldsymbol{r}_{n}^{\prime}\right)\right\rangle \\
& \propto R^{-\mu_{n}^{(p)}},
\end{aligned}
$$

where $R$ is a typical separation between any pair, and we are interested in the scaling relations between the exponents $\mu_{n}^{(p)}$ and the exponents $\zeta_{n}$. Note that $\mu_{0}^{(2)}$ in this notation is the well studied [17,12 exponent of dissipation fluctuation which is denoted $\mu$. On the basis of the Navier-Stokes equations and the fusion rules we establish rigorously that

$$
\mu_{n}^{(p)}=p-\zeta_{n+3 p}
$$

In particular we offer a solid derivation of the phenomenologically guessed bridge relation [9, 14 $\mu=2-\zeta_{6}$.

In Section VIII we summarize the paper and indicate the direction of the road ahead.

\section{FUSION RULES FOR THE INERTIAL INTERVAL}

In this section we discuss the fusion rules that arise when some of the coordinates of an $n$-point correlation function tend toward one other. We begin by introducing the set of correlation functions that are required by the analysis.

\section{A. Correlation functions and related quantities}

The fully unfused correlation function $\boldsymbol{F}_{n}$ was introduced in Eq.(1.8). Other statistical quantities of interest have the same number of velocity differences but they depend on a smaller number of coordinates. In the language developed below we will refer to such quantities as partially fused correlation functions. For example if all the velocity differences are evaluated with respect to the same point we define an $n$-rank tensor

$$
\boldsymbol{S}_{n}\left(\boldsymbol{r}_{0} \mid \boldsymbol{r}_{1}, \boldsymbol{r}_{2} \ldots \boldsymbol{r}_{n}\right)=\left\langle\boldsymbol{w}\left(\boldsymbol{r}_{0}, \boldsymbol{r}_{1}\right) \boldsymbol{w}\left(\boldsymbol{r}_{0}, \boldsymbol{r}_{2}\right) \ldots \boldsymbol{w}\left(\boldsymbol{r}_{0}, \mathbf{r}_{n}\right)\right\rangle
$$

Note that in an inhomogeneous ensemble this quantity depends explicitly on $\boldsymbol{r}_{0}$. Obviously, $\boldsymbol{F}_{n}$ depends on many more coordinates than $\boldsymbol{S}_{n}$. It is useful sometimes, therefore, to represent correlation functions $\boldsymbol{F}_{n}$ in terms of the partly fused quantities $\boldsymbol{S}_{n}$. This can always be done: represent $\boldsymbol{w}\left(\boldsymbol{r}_{j}, \boldsymbol{r}_{j}^{\prime}\right)$ as $\boldsymbol{w}\left(\boldsymbol{r}_{j}, \boldsymbol{r}_{0}\right)+\boldsymbol{w}\left(\boldsymbol{r}_{0}, \boldsymbol{r}_{j}^{\prime}\right)$, and by substitution find the needed relation. It can also be useful to choose $\boldsymbol{r}_{0}$ as the center of mass of all the coordinates in $\boldsymbol{F}_{n}$. We will return to such relations when we need them.

One can fuse more points together. For example we can have only one separation vector distance, and form the $n$-rank tensor

$$
\hat{\boldsymbol{S}}_{n}(\boldsymbol{R}) \equiv \hat{\boldsymbol{S}}_{n}\left(\boldsymbol{r}_{0} \mid \boldsymbol{r}\right)=\boldsymbol{S}_{n}\left(\boldsymbol{r}_{0} \mid \boldsymbol{r}, \boldsymbol{r} \ldots \boldsymbol{r}\right)
$$

where $\boldsymbol{R} \equiv \boldsymbol{r}-\boldsymbol{r}_{0}$. Equations (2.1) and (2.2) can be understood as the limit of (1.8) when pairs of coordinates fuse together. For example, in going from (1.8) to (2.1) we fuse all the unprimed coordinates, and keep all the primed coordinates different. To get (2.2) we also fuse all the primed coordinates.

Note that one can also consider another type of fusion, in which pairs of coordinates $\boldsymbol{r}_{j}$ and $\boldsymbol{r}_{j}^{\prime}$ which are associated with the velocity difference $\boldsymbol{w}\left(\boldsymbol{r}_{j}, \boldsymbol{r}_{j}^{\prime}\right)$ coalesce. We will refer to the fusion of coordinates with a velocity difference as a fusion of type A, and a fusion of coordinates that are not associated with a velocity difference as a fusion of type B. The properties of these two limiting procedures are different, and they have to be studied separately. It is commonly assumed that the limit of type B exists, and the limit remains finite when $\nu \rightarrow 0$. This is equivalent to the statement that $\boldsymbol{S}_{n}\left(\boldsymbol{r}_{0} \mid \boldsymbol{r}_{1}, \mathbf{r}_{2} \ldots \boldsymbol{r}_{n}\right)$ and $\boldsymbol{S}_{n}(\boldsymbol{R})$ are independent of Re for asymptotically large Re. Although this property looks innocent, it is a deep 
statement that in general needs to be supported by theory. In the language of diagrammatic perturbation theory this property is a consequence of the locality of every diagram in the perturbation expansion for these quantities; this property was discussed in detail in [2]. In [3] it was explained that there exists a mechanism for the appearance of the viscous scale in the statistical theory. However, this mechanism operates only when one correlates gradients of the velocity fields rather than the velocity differences themselves. Thus the above statement is equivalent to the assumption that there are no additional non-perturbative mechanisms for the appearance of the viscous scales in the theory of correlations of velocity differences. Additional objects that appear naturally in the theory are correlations whose tensorial nature is of lower rank, including scalars and vectors. The first one is the scalar quantity which is appropriate for even orders of $\boldsymbol{S}_{n}$. To keep in mind its scalar nature we denoted it in [4] as $\stackrel{o}{S}_{2 m}(R)$ and define it as

$$
\stackrel{o}{S}_{2 m}(\boldsymbol{R}) \equiv\left\langle\left|\boldsymbol{w}\left(\boldsymbol{r}_{0}, \boldsymbol{r}\right)\right|^{2 m}\right\rangle, \quad \boldsymbol{R} \equiv \boldsymbol{r}-\boldsymbol{r}_{0}
$$

The quantity $\stackrel{o}{S}_{2 m}(R)$ is analytic. For odd order structure functions we introduce a vector object $S_{2 m+1}^{\alpha}(\boldsymbol{R})$ according to

$$
S_{2 m+1}^{\alpha}(\boldsymbol{R}) \equiv\left\langle w_{\alpha}\left(\boldsymbol{r}_{0}, \boldsymbol{r}\right)\left|\boldsymbol{w}\left(\boldsymbol{r}_{0}, \boldsymbol{r}\right)\right|^{2 m}\right\rangle .
$$

Here and below we will use Greek indices to indicate vector and tensor components, and Roman indices to indicate the order of the quantity. The placement of indices as subscripts or superscripts has no special meaning, and is chosen for convenience.

In isotropic turbulence the vector $S_{2 m+1}^{\alpha}(\boldsymbol{R})$ can only be oriented along $\boldsymbol{R}$. This allows us the introduction of a scalar quantity $S_{2 m+1}(R)$ which depends on the magnitude of $R$ :

$$
S_{2 m+1}^{\alpha}(\boldsymbol{R})=\frac{R_{\alpha}}{R} S_{2 m+1}(R)
$$

In later sections we use the objects defined here to study the fusion rules and their consequences.

\section{B. Basic Properties of Correlation Functions in Systems with Flux Equilibrium}

The systems that we discuss are driven on a characteristic scale referred to as the outer scale $L$. This driving can be achieved by either a time dependent low frequency "stirring force" or by specifying given values of $\boldsymbol{u}$ at a set of "boundary" points with a characteristic separation $L$ away from our observation points $\boldsymbol{r}_{0}, \boldsymbol{r}_{0}^{\prime}, \boldsymbol{r}_{1}$, etc. The system suffers dissipation due to viscosity, and in the inviscid limit the kinetic energy is conserved. In three dimensions we deal with a "direct" energy cascade in which the intake of energy on the scale $L$ is balanced by dissipation on a small scale $\eta \ll L$.
We invoke two fundamental assumptions [15]:

1. Scale invariance: all the correlation functions are homogeneous functions of their arguments in the core of the inertial interval $\eta \ll\left|\boldsymbol{r}_{i}-\boldsymbol{r}_{0}\right| \ll L$ :

$$
\boldsymbol{F}_{n}\left(\lambda \boldsymbol{r}_{1}, \lambda \boldsymbol{r}_{1}^{\prime} ; \ldots ; \lambda \mathbf{r}_{n}, \lambda \boldsymbol{r}_{n}^{\prime}\right)=\lambda^{\zeta_{n}} \boldsymbol{F}_{n}\left(\mathbf{r}_{1}, \boldsymbol{r}_{1}^{\prime} ; \ldots ; \boldsymbol{r}_{n}, \boldsymbol{r}_{n}^{\prime}\right),
$$

where $\zeta_{n}$ are scaling exponents. It is obvious that all the other types of correlation and structure functions that emanate from this most general quantity and which were detailed in the previous subsection share the same scaling exponents. Accordingly $\zeta_{n}$ is also the usual scaling exponent of the $n$-th order structure function (2.3).

2. Universality of the scaling exponents: this means that we can fix an arbitrary set of velocity differences on the scale of $L$, and the scaling exponents of the correlation functions will not depend on the precise choice of the $L$-scale motions. Mathematically this is expressed as the following property of the conditional average: if for $i \leq n$ $\left|\boldsymbol{r}_{i}-\boldsymbol{r}_{i}^{\prime}\right| \ll L$, and for $i>n,\left|\boldsymbol{r}_{i}^{\prime}-\boldsymbol{r}_{i}\right| \sim L$, then

$$
\begin{aligned}
&\left\langle\boldsymbol{w}\left(\boldsymbol{r}_{1}, \boldsymbol{r}_{1}^{\prime}\right) \boldsymbol{w}\left(\mathbf{r}_{2}, \boldsymbol{r}_{2}^{\prime}\right) \ldots \boldsymbol{w}\left(\boldsymbol{r}_{n}, \boldsymbol{r}_{n}^{\prime}\right)\right| \boldsymbol{w}\left(\boldsymbol{r}_{n+1}, \mathbf{r}_{n+1}^{\prime}\right) \\
&\left.\ldots \boldsymbol{w}\left(\boldsymbol{r}_{n+N}, \boldsymbol{r}_{n+N}^{\prime}\right)\right\rangle=\tilde{\boldsymbol{F}}_{n}\left(\boldsymbol{r}_{1}, \boldsymbol{r}_{1}^{\prime} ; \ldots ; \boldsymbol{r}_{n}, \boldsymbol{r}_{n}^{\prime}\right) \\
& \times \Phi_{n, N}\left(\boldsymbol{r}_{n+1}, \boldsymbol{r}_{n+1}^{\prime} ; \ldots ; \boldsymbol{r}_{n+N}, \boldsymbol{r}_{n+N}^{\prime}\right)
\end{aligned}
$$

The precise meaning of the universality assumption is that the functions $\widetilde{\boldsymbol{F}}_{n}$ have the same scaling exponents as $\boldsymbol{F}_{n}$ in the inertial interval. They may be different functions in the inertial interval and in particular they may differ in their crossover to viscous behavior. Their (different) crossover scales may depend on the large scale motions that were fixed in the conditional average.

\section{Fusion rules for the fusion of $p$ pairs of points in velocity differences (type A)}

\section{General case: $2 \leq p \leq n-2$}

The first set of fusion rules that we derive concerns $\boldsymbol{F}_{n}$ when $p$ pairs of points $\boldsymbol{r}_{1}, \boldsymbol{r}_{1}^{\prime} \ldots \boldsymbol{r}_{p}, \boldsymbol{r}_{p}^{\prime},(p<n)$ which involve $p$ velocity differences tend to some point $\boldsymbol{r}_{0}$, see Fig. II C 1. Here we exclude the special cases $p=1$ and $p=n-1$ in which the leading scaling contribution vanishes by symmetry. We will consider these special cases in the next subsections.

Consider the situation in which the typical separation of all the coordinates $\boldsymbol{r}_{1}, \ldots, \boldsymbol{r}_{p}^{\prime}$ from $\boldsymbol{r}_{0}$ is $r$, whereas all the other separations remain much larger, say of the order of $R, r \ll R \ll L$. We will show, on the basis of assumptions $\mathbf{1}$ and $\mathbf{2}$, that

$$
\begin{aligned}
& \boldsymbol{F}_{n}\left(\boldsymbol{r}_{1}, \boldsymbol{r}_{1}^{\prime} ; \ldots ; \boldsymbol{r}_{n}, \boldsymbol{r}_{n}^{\prime}\right) \\
= & \tilde{\boldsymbol{F}}_{p}\left(\boldsymbol{r}_{1}, \boldsymbol{r}_{1}^{\prime} ; \ldots ; \boldsymbol{r}_{p}, \boldsymbol{r}_{p}^{\prime}\right) \boldsymbol{\Psi}_{n, p}\left(\boldsymbol{r}_{p+1}, \boldsymbol{r}_{p+1}^{\prime} ; \ldots ; \boldsymbol{r}_{n}, \boldsymbol{r}_{n}^{\prime}\right),
\end{aligned}
$$


where $\tilde{\boldsymbol{F}}_{p}$ is a tensor of rank $p$ associated with the first $p$ tensor indices of $\boldsymbol{F}_{n}$. The $(n-p)$-rank tensor $\boldsymbol{\Psi}_{n, p}\left(\boldsymbol{r}_{p+1}, \boldsymbol{r}_{p+1}^{\prime} ; \ldots ; \boldsymbol{r}_{n}, \mathbf{r}_{n}^{\prime}\right)$ is a homogeneous function with a scaling exponent $\zeta_{n}-\zeta_{p}$, and is associated with the remaining $n-p$ indices of $\boldsymbol{F}_{n}$. The derivation of the fusion rule (2.8) follows from Bayes' theorem. We write

$$
\begin{aligned}
& \boldsymbol{F}_{n}\left(\boldsymbol{r}_{1}, \boldsymbol{r}_{1}^{\prime} ; \ldots \boldsymbol{r}_{n}, \boldsymbol{r}_{n}^{\prime}\right)=\int d \boldsymbol{w}\left(\boldsymbol{r}_{p+1}, \boldsymbol{r}_{p+1}^{\prime}\right) \ldots d \boldsymbol{w}\left(\mathbf{r}_{n}, \boldsymbol{r}_{n}^{\prime}\right) \\
& \boldsymbol{w}\left(\boldsymbol{r}_{p+1}, \boldsymbol{r}_{p+1}^{\prime}\right) \ldots \boldsymbol{w}\left(\mathbf{r}_{n}, \boldsymbol{r}_{n}^{\prime}\right) \mathcal{P}\left[\boldsymbol{w}\left(\boldsymbol{r}_{p+1}, \boldsymbol{r}_{p+1}^{\prime}\right) \ldots \boldsymbol{w}\left(\boldsymbol{r}_{n}, \boldsymbol{r}_{n}^{\prime}\right)\right] \\
& \times\left\langle\boldsymbol{w}\left(\boldsymbol{r}_{1}, \boldsymbol{r}_{1}^{\prime}\right), \boldsymbol{w}\left(\boldsymbol{r}_{2}, \boldsymbol{r}_{2}^{\prime}\right) \ldots \boldsymbol{w}\left(\boldsymbol{r}_{p}, \boldsymbol{r}_{p}^{\prime}\right)\right| \boldsymbol{w}\left(\boldsymbol{r}_{p+1}, \boldsymbol{r}_{p+1}^{\prime}\right) \\
& \left.\times \boldsymbol{w}\left(\boldsymbol{r}_{p+2}, \boldsymbol{r}_{p+2}^{\prime}\right) \ldots \boldsymbol{w}\left(\boldsymbol{r}_{n}, \boldsymbol{r}_{n}^{\prime}\right)\right\rangle,
\end{aligned}
$$

where $\mathcal{P}\left[\boldsymbol{w}\left(\boldsymbol{r}_{p+1}, \boldsymbol{r}_{p+1}^{\prime}\right) \ldots \boldsymbol{w}\left(\boldsymbol{r}_{n}, \boldsymbol{r}_{n}^{\prime}\right)\right]$ is the probability to see the tensor $\boldsymbol{w}\left(\boldsymbol{r}_{p+1}, \boldsymbol{r}_{p+1}^{\prime}\right) \ldots \boldsymbol{w}\left(\boldsymbol{r}_{n}, \boldsymbol{r}_{n}^{\prime}\right)$. Next note the consequence of assumption 2: the scaling laws of the correlation functions at scale $r$ are the same independent of whether we force the system on the scale $L \gg r$ or on the scale $R \gg r$. The conditional average in (2.9) is proportional to $\tilde{\boldsymbol{F}}_{p}$, and hence (2.8).

FIG. II C 1 The geometry of fusion of type A. Lines connecting points indicate velocity differences across that distance. In this example there are three velocity differences across small separations (of the order of $r$ ) and two velocity differences across large separations, of the order of $R$.

The first impression is that (2.8) means statistical independence of the small scale motion from the large scales. This is not so. The difference is that the assumption of statistical independence would lead to $\boldsymbol{F}_{n}=\boldsymbol{F}_{p} \boldsymbol{F}_{n-p}$. Indeed, the first factor $\boldsymbol{F}_{p}$ has the same order of magnitude and the same exponent as $\tilde{\boldsymbol{F}}_{p}$ in 2.8. However, the factor $\boldsymbol{F}_{n-p}$ has a scaling exponent $\zeta_{n-p}$ rather than $\zeta_{n}-\zeta_{p}$ which is the exponent of $\boldsymbol{\Psi}_{n, p}$. When all the large separation are of the same order $R, \Psi_{n, p} \sim S_{n}(R) / S_{p}(R)$ which in the case of multi-scaling is much larger than $\boldsymbol{F}_{n-p} \sim S_{n-p}(R)$. We thus understand that the fusion rules in fact demonstrate the existence of a very special statistical dependence of the small scales on the large scales. This dependence stems physically from the existence of a direct energy flux from large to small scales. We will see that it can lead to a totally unconventional scaling structure of the theory. We should stress that these fusion rules were derived from first principles for $p=2$ in Navier-Stokes turbulence [4] and for passive scalar advection for any $p$ 18].

\section{Fusion of one pair of points in a velocity difference}

As we mentioned the case $p=1$ in which the velocity difference across a small scale $r \equiv\left|\boldsymbol{r}_{1}-\boldsymbol{r}_{1}^{\prime}\right|$ is correlated with $n-1$ velocity differences across larger distances requires a special attention. A naive application of the fusion rule 2.8) results in $\boldsymbol{F}_{n} \propto \boldsymbol{F}_{1}\left(\boldsymbol{r}_{1}, \boldsymbol{r}_{1}^{\prime}\right)$ which vanishes due to space homogeneity. In order to evaluate the leading non-vanishing term we expand $\boldsymbol{u}\left(\boldsymbol{r}_{1}^{\prime}\right)$ in a Taylor series around $\boldsymbol{u}\left(\boldsymbol{r}_{1}\right)$,

$$
\boldsymbol{u}\left(\boldsymbol{r}_{1}^{\prime}\right)=\boldsymbol{u}\left(\boldsymbol{r}_{1}\right)+\nabla_{1} \boldsymbol{u}\left(\boldsymbol{r}_{1}\right) \cdot\left(\boldsymbol{r}_{1}^{\prime}-\boldsymbol{r}_{1}\right)+\ldots .
$$

Using this we can write

$$
\begin{aligned}
& \lim _{r_{1} \rightarrow r_{1}^{\prime}} \boldsymbol{F}_{n}\left(\boldsymbol{r}_{1}, \boldsymbol{r}_{1}^{\prime} ; \ldots ; \mathbf{r}_{n}, \boldsymbol{r}_{n}^{\prime}\right) \\
& =\left(\boldsymbol{r}_{1}^{\prime}-\boldsymbol{r}_{1}\right) \cdot \nabla_{1}\left\langle\boldsymbol{u}\left(\boldsymbol{r}_{1}\right) \boldsymbol{w}\left(\mathbf{r}_{2}, \boldsymbol{r}_{2}^{\prime}\right) \ldots \boldsymbol{w}\left(\boldsymbol{r}_{n}, \boldsymbol{r}_{n}^{\prime}\right)\right\rangle .
\end{aligned}
$$

The correlation function in this formula depends an all the separation distances, and the gradient with respect to $\boldsymbol{r}_{1}$ picks up contributions from all the differences $\boldsymbol{r}_{j}-\boldsymbol{r}_{1}$. Therefore the gradient can be evaluated as the inverse of the smallest of these, $\left|\nabla_{i}\right| \sim 1 / R_{\min }$ where $R_{\text {min }} \equiv \min _{i}\left\{\left|\boldsymbol{r}_{i}-\boldsymbol{r}_{1}\right|\right\}$. For $r \ll R_{\text {min }}$ this leads to the evaluation

$$
\boldsymbol{F}_{n}\left(\boldsymbol{r}_{1}, \boldsymbol{r}_{1}^{\prime} ; \ldots ; \boldsymbol{r}_{n}, \boldsymbol{r}_{n}^{\prime}\right) \sim \frac{r}{R_{\min }} S_{n}(R) .
$$

This formula creates an immediate worry about the situation in which $R_{\min }$ becomes of the order of $r$ or smaller. We will analyze this situation in Subsection A. Now we will consider the next special case of fusion, when $p=n-1$.

\section{Fusion rule for one large separation distance}

Consider $\boldsymbol{F}_{n}\left(\boldsymbol{r}_{1}, \boldsymbol{r}_{1}^{\prime} ; \boldsymbol{r}_{2}, \boldsymbol{r}_{2}^{\prime} ; \ldots ; \boldsymbol{r}_{n}, \boldsymbol{r}_{n}^{\prime}\right)$ with all the coordinates being nearby except for $\boldsymbol{r}_{1}$ which is far away, a distance $R$ from the remaining $2 n-1$ coordinates which are all within a ball of radius $r$. Since we assume that the flow is isotropic, the tensor $\boldsymbol{F}_{n}$ is a (generally reducible) representation of the rotation group. Moreover, in the situation discussed here the isotropization of the small scales (of the order of $r$ ) with respect to the direction of $\boldsymbol{R} \equiv \boldsymbol{r}_{1}^{\prime}-\boldsymbol{r}_{1}$ leads to a direct product structure

$$
\begin{aligned}
& F_{n}^{\alpha_{1} \alpha_{2} \ldots \alpha_{n}}\left(\boldsymbol{r}_{1}, \boldsymbol{r}_{1}^{\prime} ; \boldsymbol{r}_{2}, \mathbf{r}_{2}^{\prime} ; \ldots ; \boldsymbol{r}_{n}, \boldsymbol{r}_{n}^{\prime}\right) \\
= & C \frac{R^{\alpha_{1}}}{R} \tilde{F}_{n}^{\alpha_{2} \ldots \alpha_{n}}\left(R, \boldsymbol{r}_{2}, \boldsymbol{r}_{2}^{\prime} ; \ldots ; \boldsymbol{r}_{n}, \boldsymbol{r}_{n}^{\prime}\right) .
\end{aligned}
$$

The constraint of incompressibility, which is always true, can be written as

$$
\nabla_{1}^{\alpha_{1}} F_{n}^{\alpha_{1} \alpha_{2} \ldots \alpha_{n}}\left(\boldsymbol{r}_{1}, \mathbf{r}_{1}^{\prime} ; \boldsymbol{r}_{2}, \boldsymbol{r}_{2}^{\prime} ; \ldots ; \boldsymbol{r}_{n}, \boldsymbol{r}_{n}^{\prime}\right)=0 .
$$

Applying the divergence operator to (2.12) we find the following form of the incompressibility constraint.

$$
C\left(2+R \frac{\partial}{\partial R}\right) \tilde{F}_{n}^{\alpha_{2} \ldots \alpha_{n}}\left(R, \boldsymbol{r}_{2}, \boldsymbol{r}_{2}^{\prime} ; \ldots ; \boldsymbol{r}_{n}, \boldsymbol{r}_{n}^{\prime}\right)=0
$$

This equation has two solutions:(i) $C=0$, (ii) $\tilde{F}_{n} \propto$ $1 / R^{2}$. However according to the general rule $\tilde{F}_{n}$ must be 
proportional to $R^{\zeta_{n}-\zeta_{n-1}}$. Thus the first solution is realized, the contribution which is $\propto R^{\zeta_{n}-\zeta_{n-1}}$ vanishes due to the incompressibility constraint. Therefore we need to consider the next non-vanishing evaluation which is $R$-independent:

$$
F_{n}^{\alpha_{1} \alpha_{2} \ldots \alpha_{n}}\left(\boldsymbol{r}_{1}, \boldsymbol{r}_{1}^{\prime} ; \boldsymbol{r}_{2}, \mathbf{r}_{2}^{\prime} ; \ldots ; \boldsymbol{r}_{n}, \boldsymbol{r}_{n}^{\prime}\right) \propto r^{\zeta_{n}} .
$$

This result is interesting. It means that in the case considered here in which all the separations of order $r$ except one (which is of order $R, \eta \ll r \ll R \ll L$ ) the scaling exponent of the correlation function $F_{n}$ is fully determined by small $r$-scale fluctuations.

In addition to these cases one needs some special geometries of fusions of type $\mathrm{A}$ in which the general evaluation is inapplicable. These cases are treated in Appendix A and referred to as needed.

\section{Rules for Fusions of Type B}

FIG. IID The geometry of fusion of type B. Lines connecting points indicate velocity differences across that distance. In this example there are three velocity differences across large separations, but three coordinates within a ball of small radius $r$.

\section{Coalescence of two points $(p=2)$}

Fusions of type B refer to situations in which there are $p$ coordinates within a ball of small radius $r$, but no two coordinates that belong to a velocity difference, see Fig IID. The simplest case is $p=2$ in which $\left|\boldsymbol{r}_{1}-\boldsymbol{r}_{2}\right|$ is much smaller than any other separation. Consider the velocity differences $\boldsymbol{w}\left(\boldsymbol{r}_{1}, \boldsymbol{r}_{1}^{\prime}\right)$ and $\boldsymbol{w}\left(\boldsymbol{r}_{2}, \boldsymbol{r}_{2}^{\prime}\right)$. They can be reexpressed as

$$
\begin{aligned}
& \boldsymbol{w}\left(\boldsymbol{r}_{1}, \boldsymbol{r}_{1}^{\prime}\right)=\boldsymbol{w}\left(\boldsymbol{r}_{1}, \boldsymbol{r}_{0}\right)+\boldsymbol{w}\left(\boldsymbol{r}_{0}, \boldsymbol{r}_{1}^{\prime}\right) \\
& \boldsymbol{w}\left(\boldsymbol{r}_{2}, \boldsymbol{r}_{2}^{\prime}\right)=\boldsymbol{w}\left(\boldsymbol{r}_{0}, \boldsymbol{r}_{2}^{\prime}\right)+\boldsymbol{w}\left(\boldsymbol{r}_{2}, \boldsymbol{r}_{0}\right)
\end{aligned}
$$

where $\boldsymbol{r}_{0}=\left[\boldsymbol{r}_{1}+\boldsymbol{r}_{2}\right] / 2$. This allows us to write

$\left\langle\boldsymbol{w}\left(\boldsymbol{r}_{1}, \boldsymbol{r}_{1}^{\prime}\right) \boldsymbol{w}\left(\boldsymbol{r}_{2}, \boldsymbol{r}_{2}^{\prime}\right)\{w\}^{n-2}\right\rangle=\left\langle\boldsymbol{w}\left(\boldsymbol{r}_{1}^{\prime}, \boldsymbol{r}_{0}\right) \boldsymbol{w}\left(\boldsymbol{r}_{2}^{\prime}, \boldsymbol{r}_{0}\right)\{w\}^{n-2}\right\rangle$

$-\left\langle\boldsymbol{w}\left(\boldsymbol{r}_{1}, \boldsymbol{r}_{0}\right) \boldsymbol{w}\left(\boldsymbol{r}_{2}, \boldsymbol{r}_{0}\right)\{w\}^{n-2}\right\rangle-\left\langle\boldsymbol{w}\left(\boldsymbol{r}_{1}^{\prime}, \boldsymbol{r}_{0}\right) \boldsymbol{w}\left(\boldsymbol{r}_{2}, \boldsymbol{r}_{0}\right)\{w\}^{n-2}\right\rangle$

$+\left\langle\boldsymbol{w}\left(\boldsymbol{r}_{1}, \boldsymbol{r}_{0}\right) \boldsymbol{w}\left(\boldsymbol{r}_{2}, \boldsymbol{r}_{0}\right)\{w\}^{n-2}\right\rangle$.

Here we used the short-hand notation $\{w\}^{n-2}$ to denote the remaining product of $n-2$ velocity differences.

The first term on the RHS is independent of the small separation, and is a homogeneous function of the large separations with a scaling exponent $\zeta_{n}$. The next two terms contain one velocity difference across small separations which according to the discussion of Fig. is proportional to $r^{\zeta_{2}}$. The last term has two velocity differences across a small separation, and is also proportional to the same factor. This allows one to formulate a fusion rule of type B for fusion of two points (not associated with velocity difference) in the following form:

$$
\begin{aligned}
& \boldsymbol{F}_{n}\left(\boldsymbol{r}_{1}, \boldsymbol{r}_{1}^{\prime} ; \boldsymbol{r}_{2}, \boldsymbol{r}_{2}^{\prime} ;\left\{\boldsymbol{r}_{k}, \boldsymbol{r}_{k}^{\prime}\right\}\right)-\boldsymbol{F}_{n}\left(\boldsymbol{r}_{1}, \boldsymbol{r}_{1}^{\prime} ; \boldsymbol{r}_{1}, \boldsymbol{r}_{2}^{\prime} ;\left\{\boldsymbol{r}_{k}, \boldsymbol{r}_{k}^{\prime}\right\}\right) \\
& =\tilde{\boldsymbol{S}}_{2}\left(\left|\boldsymbol{r}_{1}-\boldsymbol{r}_{2}\right|\right) \boldsymbol{\Psi}_{n, 2}\left(\boldsymbol{r}_{0} ; \boldsymbol{r}_{1}^{\prime}, \boldsymbol{r}_{2}^{\prime} ;\left\{\boldsymbol{r}_{k}, \boldsymbol{r}_{k}^{\prime}\right\}\right) \propto\left(\frac{r}{R}\right)^{\zeta_{2}} R^{\zeta_{n}} .
\end{aligned}
$$

\section{Coalescence of three points $(p=3)$}

The next topic of discussion is the fusion of type B (without velocity differences) of three points, (say $\boldsymbol{r}_{1}, \boldsymbol{r}_{2}$, and $\boldsymbol{r}_{3}$ ), as shown in Fig. IID. As before the separations between these points are all of the order of $r$, and all the other separations are much larger, of the order of $R$, $R \gg r$. As in the case of $p=2$ we denote the center of mass of these coordinates as $\boldsymbol{r}_{0}: \boldsymbol{r}_{0}=\left(\boldsymbol{r}_{1}+\boldsymbol{r}_{2}+\boldsymbol{r}_{3}\right) / 3$. We express the two of the velocity differences according to (2.16), and the third velocity difference in analogy:

$$
\boldsymbol{w}\left(\boldsymbol{r}_{3}, \boldsymbol{r}_{3}^{\prime}\right)=\boldsymbol{w}\left(\boldsymbol{r}_{3}, \boldsymbol{r}_{0}\right)+\boldsymbol{w}\left(\boldsymbol{r}_{0}, \boldsymbol{r}_{3}^{\prime}\right)
$$

Clearly, the correlation function

$$
\begin{aligned}
& \boldsymbol{F}_{n}\left(\boldsymbol{r}_{1}, \boldsymbol{r}_{1}^{\prime} ; \boldsymbol{r}_{2}, \boldsymbol{r}_{2}^{\prime} ; \boldsymbol{r}_{3}, \boldsymbol{r}_{3}^{\prime} ;\left\{\boldsymbol{r}_{k}, \boldsymbol{r}_{k}^{\prime}\right\}\right) \\
& =\left\langle\boldsymbol{w}\left(\boldsymbol{r}_{1}, \boldsymbol{r}_{1}^{\prime}\right) \boldsymbol{w}\left(\boldsymbol{r}_{2}, \boldsymbol{r}_{2}^{\prime}\right) \boldsymbol{w}\left(\boldsymbol{r}_{3}, \boldsymbol{r}_{3}^{\prime}\right)\{w\}^{n-3}\right\rangle
\end{aligned}
$$

has three types of contributions. The first, $\boldsymbol{F}_{n}^{(0)}$, is independent of the small separations,

$$
\boldsymbol{F}_{n}^{(0)}=\boldsymbol{F}\left(\boldsymbol{r}_{0}, \boldsymbol{r}_{1}^{\prime} ; \boldsymbol{r}_{0}, \boldsymbol{r}_{2}^{\prime} ; \boldsymbol{r}_{0}, \boldsymbol{r}_{3}^{\prime} ;\left\{\boldsymbol{r}_{k}, \boldsymbol{r}_{k}^{\prime}\right\}\right)
$$

The second type of contribution has either one velocity difference or a product of two velocity differences across a small separation. Both these contributions have a leading term that is proportional to $r^{\zeta_{2}}$. The third type of contribution has a product of three velocity differences across a small separation and is proportional to $r^{\zeta_{3}}$. In conclusion, the scaling behavior is similar to the one displayed in Eq.(A8), with the addition of a term constant in $r, \boldsymbol{F}_{n}^{(0)}$.

\section{General case: fusion of $n$ points without velocity difference}

The above discussion of fusion of type B of two and three points allows us to offer a general statement concerning the asymptotic behavior of $n$-point correlation functions $F_{n}$ when $p$ of the coordinates are separated from each other by small distances of the order of $r$, and $2 n-p$ coordinates are separated by a large separation of the order of $R$. In terms of the definition (1.8) we will take the $p$ close-by coordinates as $\boldsymbol{r}_{1}, \boldsymbol{r}_{2}, \ldots, \boldsymbol{r}_{p}$, and the 
$2 n-p$ coordinates as $\boldsymbol{r}_{p+1}, \ldots, \boldsymbol{r}_{n}$ and $\boldsymbol{r}_{1}^{\prime}, \ldots, \boldsymbol{r}_{n}^{\prime}$. Recall that this choice means that we do not have velocity differences across small scales.

To see the asymptotic behavior we need to repeat the substitutions of the type (2.16) and (2.19), i.e. for $1 \leq j \leq p$

$$
\boldsymbol{w}\left(\boldsymbol{r}_{j}, \boldsymbol{r}_{j^{\prime}}^{\prime}\right)=\boldsymbol{w}\left(\boldsymbol{r}_{j}, \boldsymbol{r}_{0}\right)+\boldsymbol{w}\left(\boldsymbol{r}_{0}, \boldsymbol{r}_{j^{\prime}}^{\prime}\right) .
$$

As before the center of mass is $\boldsymbol{r}_{0}=\left(\sum_{k=1}^{p} \boldsymbol{r}_{k}\right) / p$. The result of this substitution can be readily guessed from the cases $p=2,3$ :

$$
\boldsymbol{F}_{n}\left(\boldsymbol{r}_{1}, \boldsymbol{r}_{1}^{\prime} ; \ldots ; \boldsymbol{r}_{p}, \boldsymbol{r}_{p}^{\prime} ;\left\{\boldsymbol{r}_{k}, \boldsymbol{r}_{k}^{\prime}\right\}\right)=\boldsymbol{F}_{n, p}^{(0)}+\sum_{j=2}^{p} \boldsymbol{F}_{n, p}^{(j)}
$$

In this formula $\boldsymbol{F}_{n, p}^{(0)}$ is independent of the small separation, and each term $\boldsymbol{F}_{n, p}^{(j)}$ originates from $j$ velocity differences across small separations. These velocity differences are $\boldsymbol{w}\left(\boldsymbol{r}_{i}, \boldsymbol{r}_{0}\right)$ where $i \leq p$. There are always $C_{p}^{j}=p ! / j !(p-j)$ ! different products of $j$ velocity differences across small scales in $\boldsymbol{F}_{n, p}^{(j)}$. More explicitly,

$$
\begin{aligned}
\boldsymbol{F}_{n, p}^{(2)} & =\left[\sum_{i_{1}>i_{2}=1}^{p} S_{2}\left(\boldsymbol{r}_{0} \mid \boldsymbol{r}_{i_{1}}, \boldsymbol{r}_{i_{2}}\right)\right] \boldsymbol{\Psi}_{n, 2}, \\
\boldsymbol{F}_{n, p}^{(3)} & =\left[\sum_{i_{1}>i_{2}>i_{3}=1}^{p} \tilde{\boldsymbol{S}}_{3}\left(\boldsymbol{r}_{0} \mid \boldsymbol{r}_{i_{1}}, \boldsymbol{r}_{i_{2}}, \boldsymbol{r}_{i_{3}}\right)\right] \boldsymbol{\Psi}_{n, 3},
\end{aligned}
$$

etc. On the LHS of these equations the functions $\boldsymbol{F}_{n, p}^{(j)}$ depend on all the coordinates. The function $\tilde{\boldsymbol{S}}_{j}$ is a homogeneous function of $j$ separations $\boldsymbol{r}_{i}-\boldsymbol{r}_{0}$, with a scaling exponent $\zeta_{j}$. The functions $\boldsymbol{\Psi}_{n, j}$ are homogeneous functions of $n-p$ large separations $\boldsymbol{r}_{k}-\boldsymbol{r}_{0}$ (with $k=p+1, \ldots, n$ and $n$ large separations $\boldsymbol{r}_{m}^{\prime}-\boldsymbol{r}_{0}$ (with $m=1, \ldots, n)$ with a scaling exponent $\zeta_{n}-\zeta_{j}$. Schematically we can summarize the scaling behavior of $\boldsymbol{F}_{n, p}^{(j)}$ as

$$
\boldsymbol{F}_{n, p}^{(j)} \propto\left(\frac{r}{R}\right)^{\zeta_{j}} R^{\zeta_{n}}
$$

We stress that since we are concerned with the limit $r \ll R$, the leading term in (2.23) is always $\boldsymbol{F}_{n, p}^{(0)}$ which is independent of $r$. The leading $r$ dependence is carried by $\boldsymbol{F}_{n, p}^{(2)} \propto r^{\zeta_{2}}$. This does not mean however that the higher order terms in (2.23) are unimportant. They will provide the leading order contributions to correlation functions of velocity gradients as will be shown in subsection $2 \mathrm{G}$.

\section{E. Mixture of A- and B-type fusions}

FIG. IIE The geometry of fusion with a mixture of type A and $\mathrm{B}$

In this Subsection we consider the even more general case in which we have within the ball of small radius $r q$ pairs of points associated with the velocity differences $\boldsymbol{w}\left(\boldsymbol{r}_{i}, \boldsymbol{r}_{i}^{\prime}\right)(1 \leq i \leq q)$, and $(p-q)$ points (say $\left.\boldsymbol{r}_{q+1}, \ldots, \boldsymbol{r}_{p}\right)$ associated with velocity differences across large separations of order $R \gg r$, see Fig IIE. The rest of the coordinates $\boldsymbol{r}_{k}, \boldsymbol{r}_{k}^{\prime}, k>p$ are separated by large distances of the order of $R$, see Fig.6. For this case we need again to reexpress $(p-q)$ velocity differences $\boldsymbol{w}\left(\boldsymbol{r}_{j}, \boldsymbol{r}_{j}^{\prime}\right)$ (for $j=q+1, \ldots, p)$ in the manner of (2.22), and to substitute these into the definition of $\boldsymbol{F}_{n}(1.8)$. The result of this process is an equation similar to (2.23), but with important differences:

$$
\boldsymbol{F}_{n}\left(\boldsymbol{r}_{1}, \boldsymbol{r}_{1}^{\prime} ; \ldots ; \boldsymbol{r}_{p}, \boldsymbol{r}_{p}^{\prime} ;\left\{\boldsymbol{r}_{k}, \boldsymbol{r}_{k}^{\prime}\right\}\right)=\sum_{j=q}^{p} \tilde{\boldsymbol{F}}_{n, p}^{(j)} .
$$

There is no constant term now, and the leading order contribution is proportional to $r^{\zeta_{q}}$. The exception (as always) is that when $q=1$ the leading scaling behavior is $r^{\zeta_{2}}$. We note that the functions $\tilde{\boldsymbol{F}}_{n, p}^{(j)}$ are different from the analogous functions $\boldsymbol{F}_{n, p}^{(j)}$, but they have the same scaling behavior, $\tilde{\boldsymbol{F}}_{n, p}^{(j)} \propto R^{\zeta_{n}}(r / R)^{\zeta_{j}}$. The explicit expressions analogous to (2.24, 2.25) can be written down when needed. We will find that the subleading terms contribute the most important contributions in various situations.

\section{F. Fusion rules for the fusion of two or more groups of pairs}

The next set of fusion rules is obtained for the structure function $\boldsymbol{F}_{n}$ when two groups of $p$ and $q$ points (with $p+q<n)$ tend to $\boldsymbol{r}_{0}$ and $\boldsymbol{r}_{0}^{\prime}$ respectively. The separation between these groups of points is of the order of $R$. The derivation of the fusion rules of type $\mathrm{A}$ for the simplest situation when all the coordinates are different (and separated by $r$ or by $R$ ) obviously follows from the same basic properties of velocity correlation functions which we discussed in Subsection 2B. The result looks similar to Eq. (2.8):

$$
\begin{aligned}
& \boldsymbol{F}_{n}\left(\boldsymbol{r}_{1}, \boldsymbol{r}_{1}^{\prime} ; \ldots ; \boldsymbol{r}_{n}, \boldsymbol{r}_{n}^{\prime}\right) \\
= & \tilde{\boldsymbol{F}}_{p}\left(\boldsymbol{r}_{1}, \boldsymbol{r}_{1}^{\prime} ; \ldots ; \boldsymbol{r}_{p}, \boldsymbol{r}_{p}^{\prime}\right) \tilde{\boldsymbol{F}}_{q}\left(\boldsymbol{r}_{p+1}, \boldsymbol{r}_{p+1}^{\prime} ; \ldots ; \boldsymbol{r}_{p+q}, \mathbf{r}_{p+q}^{\prime}\right) \\
\times & \boldsymbol{\Psi}_{n, p, q}\left(\boldsymbol{r}_{p+q+1}, \boldsymbol{r}_{p+q+1}^{\prime} ; \ldots ; \boldsymbol{r}_{n}, \boldsymbol{r}_{n}^{\prime}\right) .
\end{aligned}
$$

The scaling exponent of $\boldsymbol{\Psi}_{n, p, q}$ is $\zeta_{n}-\zeta_{p}-\zeta_{q}$. As in the case of the fusion rules (2.8), also (2.28) are not decompositions into products of lower order correlation functions, and the functions $\boldsymbol{\Psi}$ are not correlations of velocity differences across large separations. As before the functions $\boldsymbol{\Psi}$ are much larger than the corresponding correla- 
tion functions in all situations with multi-scaling. Evidently one can derive similar fusion rules for three, four or more groups of coalescing points with large separations between the groups. The structure of the resulting correlation function will be a product of the correlation function associated with each group times some function $\boldsymbol{\Psi}$ of big separations which carries the overall exponent.

The generalization of these fusion rules (which are of type A) to the more complicated cases with fusions of type B or to mixed types of fusion is now obvious: we can consider every group of point separately in the way that we discussed for the case of fusion of just one group of points.

\section{G. Fusion rules for correlation functions including unfused velocity gradients}

In this subsection we use the fusion rules obtained above to evaluate the leading order scaling behavior of correlation functions that include unfused velocity derivatives. To be specific, consider the $q$-order derivatives $\boldsymbol{\nabla}_{1} \boldsymbol{\nabla}_{2} \ldots \boldsymbol{\nabla}_{q}$, with $\boldsymbol{\nabla}_{j} \equiv \partial / \partial \boldsymbol{r}_{j}$. We are going to apply this $q$-order derivative on correlation functions with $p$ fusing points, with $q \leq p$, such that the derivatives operate only on coordinates within this group of $p$ points. We will also consider a constrained derivative, i.e. such that $\nabla_{1}+\nabla_{2}+\ldots+\nabla_{q}=0$. With such a derivative we get a particularly simple result. There are two situations to consider. If the $p$ fusing points undergo a fusion of type $\mathrm{A}$, the leading scaling behavior of the $q$ order derivative is simply $r^{\zeta_{p}-q}$. In the case of fusions of type B we consult with Eq. (2.23) and find that the leading order contribution with respect to the small distance $r$ is

$$
\nabla_{1} \ldots \nabla_{q} \boldsymbol{F}_{n}=\nabla_{1} \ldots \nabla_{q} \tilde{\boldsymbol{S}}_{q}\left(\boldsymbol{r}_{0} \mid \boldsymbol{r}_{1}, \boldsymbol{r}_{2}, \ldots, \boldsymbol{r}_{q}\right) \boldsymbol{\Psi}_{n, q} .
$$

The contributions arising from the terms $\boldsymbol{F}_{n}^{(j)}$ all vanish under the derivatives. To see that this is so for $2 \leq j \leq q$ we recall (cf. (2.24), 2.25)) that although the functions $\boldsymbol{F}_{n}^{(j)}$ depend on all the $p$ separations $\boldsymbol{r}_{i}-\boldsymbol{r}_{0}$, it is a sum of functions $\tilde{S}_{j}$ each of which depends only on a subset of $j$ small separations. Thus we find a nonzero contribution for the $q$-order derivative only from terms with $j \geq q$. The leading contribution for $r \ll R$ always comes from the $j=q$ term. It is noteworthy that this contribution is independent of the remaining $p-q$ small separations, if they exist.

In summary, the rule is that when we fuse $p$ coordinates on which $q$ gradients are applied, the correlation function scales asymptotically like $r^{\zeta_{p}-q} R^{\zeta_{n}-\zeta_{p}}$ for fusions of type $\mathrm{A}$ and $r^{\zeta_{q}-q} R^{\zeta_{n}-\zeta_{q}}$ for fusions of type B. The second result is independent of the number of additional points in the ball of size $r$ that do not have a gradient applied to them. These additional points can be even fused together or with $\boldsymbol{r}_{0}$. This result will be the starting point for the discussion of the correlation function $\mathcal{J}_{n}$ of the balance equation.

\section{THE FUSION OF TWO POINTS: TENSOR STRUCTURE AND THE EFFECTS OF ANISOTROPY}

In the previous section we focused on the scaling exponents that characterize the leading contribution to the correlation function in the asymptotic regime when some points fused together. In this section we address the tensor structure of the correlation functions, and the subleading terms that exist because of the dependence on the angles between the small separation vector and the remaining large separation vectors. This second subject is related to the rich and important issue of the decay of the effects of anisotropic forcing, and we do not exhaust this issue in the present section. Some of the results are relevant however in a much broader context.

\section{A. Tensor structure in the fusion of two points}

In all the previous discussion of the fusion rules when two points (say $\boldsymbol{r}_{1}$ and $\boldsymbol{r}_{2}$ ) were fused we focused only on the scaling exponent of the function $\tilde{\boldsymbol{F}}_{2}(\boldsymbol{\rho})$ with $\boldsymbol{\rho}=\boldsymbol{r}_{1}-\boldsymbol{r}_{2}$. Here we will go further in examining the structure of the resulting correlation functions.

The fusion of two points involves just one small separation distance $\boldsymbol{\rho}=\boldsymbol{r}_{1}-\boldsymbol{r}_{2}$. In general the asymptotic behavior of the correlation function may depend on the angle between $\boldsymbol{\rho}$ and the remaining large separation vectors. This dependence is discussed in the next subsection. Here we consider the isotropic part which will be shown to be the leading contribution. It is easy to determine the dependence of $\tilde{\boldsymbol{F}}_{2}$ on the direction of $\boldsymbol{\rho}$ using the general requirement of incompressibility:

$$
\tilde{F}_{2}^{\alpha \beta}(\boldsymbol{\rho})=A\left[\left(2+\zeta_{2}\right) \delta_{\alpha \beta}-\zeta_{2} \frac{\rho_{\alpha} \rho_{\beta}}{\rho^{2}}\right] \rho^{\zeta_{2}} .
$$

This form is standard for the second-order structure function in isotropic turbulence. We reiterate that when we extract $\tilde{\boldsymbol{F}}_{2}$ out of a many-point correlation function in the process of fusion, there is the issue of the direction of $\boldsymbol{\rho}$ with respect to other vector separations which we address next.

\section{Subleading contributions: effect of helicity and anisotropy}

The dependence on the angle of $\boldsymbol{\rho}$ is an interesting subject that deserves full analysis in a separate study. Here we only touch on some of the essential issues.

The analysis of these terms depends very much on the nature of the two-point scalar correlation function 
$F_{2}(\boldsymbol{\rho}) \equiv F_{2}^{\alpha \alpha}(\boldsymbol{\rho})$ in anisotropic turbulence. When the forcing of turbulence is isotropic, this function depends on the magnitude $|\boldsymbol{\rho}|$ only. In general however the dependence on the orientation of $\boldsymbol{\rho}$ with respect to the anisotropic forces may be important. It is useful therefore to represent $F_{2}(\boldsymbol{\rho})$ as a "multipole" expansion according to

$$
\begin{aligned}
F_{2}(\boldsymbol{\rho}) & =\sum_{\ell=0}^{\infty} F_{2, \ell}(\boldsymbol{\rho}) \\
F_{2, \ell}(\boldsymbol{\rho}) & =\sum_{m=-\ell}^{\ell} Y_{\ell m}(\hat{\boldsymbol{\rho}}) \int F_{2}(\rho \boldsymbol{\xi}) Y_{\ell m}(\hat{\boldsymbol{\xi}}) d \hat{\boldsymbol{\xi}} .
\end{aligned}
$$

In this expansion the $\hat{\boldsymbol{\rho}} \equiv \boldsymbol{\rho} / \rho, \hat{\boldsymbol{\xi}}$ is unit vector and the functions $Y_{\ell m}$ are the standard spherical harmonics. We chose to expand in these functions since the relevant symmetry group in our problem is the group of rotations $\mathrm{SO}(3)$. In a scale invariant situation we expect that each component $F_{2, \ell}$ scales like

$$
F_{2, \ell} \propto \rho^{\beta_{\ell}}
$$

and in general the exponents $\beta_{\ell}$ depend on $\ell$. That this is so with universal exponents $\beta_{l}$ was proved 18] in the case of Kraichnan's model of passive scalar [19], but there is yet no analogous proof in the case of Navier-Stokes turbulence. We will assume, in order to proceed, that the exponents $\beta_{\ell}$ exist and that they are universal.

Under these assumption the calculation of the subleading contributions to the fusion rules in the case of the fusion of two points is straightforward. We first consider the partial trace $\boldsymbol{F}_{n}^{\alpha \alpha}$ of the $n$-rank tensor $\boldsymbol{F}_{n}$ with respect to the first two indices. Next we decompose it into spherical harmonics according to the "multipole" expansion [7]:

$$
\begin{aligned}
& \boldsymbol{F}_{n}^{\alpha \alpha}\left(\boldsymbol{r}_{0}+\frac{1}{2} \boldsymbol{\rho}, \boldsymbol{r}_{0}-\frac{1}{2} \boldsymbol{\rho} ;\left\{\boldsymbol{r}_{k}, \boldsymbol{r}_{k}^{\prime}\right\}\right) \\
= & \sum_{\ell=0}^{\infty} \boldsymbol{F}_{n, \ell}^{\alpha \alpha}\left(\boldsymbol{r}_{0}+\frac{1}{2} \boldsymbol{\rho}, \boldsymbol{r}_{0}-\frac{1}{2} \boldsymbol{\rho} ;\left\{\boldsymbol{r}_{k}, \boldsymbol{r}_{k}^{\prime}\right\}\right), \\
& \boldsymbol{F}_{n, \ell}^{\alpha \alpha}\left(\boldsymbol{r}_{0}+\frac{1}{2} \boldsymbol{\rho}, \boldsymbol{r}_{0}-\frac{1}{2} \boldsymbol{\rho} ;\left\{\boldsymbol{r}_{k}, \boldsymbol{r}_{k}^{\prime}\right\}\right)=\sum_{m=-\ell}^{\ell} Y_{\ell m}(\hat{\boldsymbol{\rho}}) \\
\times & \int \boldsymbol{F}_{n}^{\alpha \alpha}\left(\boldsymbol{r}_{0}+\frac{1}{2} \rho \boldsymbol{\xi}, \boldsymbol{r}_{0}-\frac{1}{2} \boldsymbol{\rho} \boldsymbol{\xi} ;\left\{\boldsymbol{r}_{k}, \boldsymbol{r}_{k}^{\prime}\right\}\right) Y_{\ell m}(\hat{\boldsymbol{\xi}}) d \hat{\boldsymbol{\xi}},
\end{aligned}
$$

The first term, $\boldsymbol{F}_{n, 0}^{\alpha \alpha}$, corresponds to the leading contribution with the scaling behavior $\rho_{2}^{\zeta} R^{\left(\zeta_{n}-\zeta_{2}\right)}$ which was discussed above. (Remember that $R$ is the characteristic separation in the correlation function $\boldsymbol{F}_{n}^{\alpha \alpha}$ ). The next order contributions are given by $\boldsymbol{F}_{n, \ell>0}^{\alpha \alpha}$

$$
\begin{aligned}
& \boldsymbol{F}_{n, \ell}^{\alpha \alpha}\left(\boldsymbol{r}_{1}, \boldsymbol{r}_{1}^{\prime} ; \boldsymbol{r}_{2}, \boldsymbol{r}_{2}^{\prime} ;\left\{\boldsymbol{r}_{k}, \boldsymbol{r}_{k}^{\prime}\right\}\right) \\
& =\tilde{F}_{2, \ell}(\boldsymbol{\rho}) \Psi_{n, 2, \ell}\left(\boldsymbol{r}_{0}, ; \boldsymbol{r}_{1}^{\prime}, \boldsymbol{r}_{2}^{\prime} ;\left\{\boldsymbol{r}_{k}, \boldsymbol{r}_{k}^{\prime}\right\}\right) .
\end{aligned}
$$

The scaling behavior of $\tilde{F}_{2, \ell}$ can be read from (3.4) under the usual assumption of universality; the two points that fuse together relate to the unfused points in the same way that the two-point correlation function relates to the anisotropic forcing. We can thus write with impunity:

$$
\tilde{F}_{2, \ell} \propto \rho^{\beta_{\ell}}, \quad \Psi_{n, 2, \ell} \propto R^{\zeta_{n}-\beta_{\ell}} .
$$

To estimate the value of $\beta_{1}$ and $\beta_{2}$ we need to understand what is the physics that determines them. In fact, since we considered the partial trace of the correlation function, we end up with $\tilde{F}_{2}$ which is even under the transformation $\boldsymbol{\rho} \rightarrow-\boldsymbol{\rho}$. Accordingly, although the exponents are as stated in (3.8) the coefficients of all odd $\ell$ quantities are zero. In order to examine odd $\ell$ contributions one needs to form a correlator which is not even in $\boldsymbol{\rho}$. As an example we consider

$$
F_{2}^{\alpha \beta}(\boldsymbol{\rho})=\left\langle u_{\alpha}(\boldsymbol{r}+\boldsymbol{\rho}) u_{\beta}(\boldsymbol{r})-u_{\alpha}(\boldsymbol{r}-\boldsymbol{\rho}) u_{\beta}(\boldsymbol{r})\right\rangle .
$$

Since this object is manifestly odd in $\boldsymbol{\rho}$ it vanishes when there exists inversion symmetry. For turbulence with non-zero helicity $F_{2}^{\alpha \beta}(\boldsymbol{\rho}) \neq 0$ and the leading contribution to $F_{2}^{\alpha \beta}(\boldsymbol{\rho})$ (which is $F_{2,1}^{\alpha \beta}(\boldsymbol{\rho})$ ) is determined by the flux of helicity. This is reminiscent of the flux of energy which determines the leading contribution to the second order structure function. Standard K41 dimensional reasoning leads to the prediction $\beta_{1}=1$, see for example [11. This holds probably with the same accuracy as the K41 prediction for $\zeta_{2}$, which is $\zeta_{2}=2 / 3$ instead of the experimental value $\zeta_{2} \approx 0.7012,13$.

We can easily determine the tensor structure of $F_{2,1}^{\alpha \beta}(\boldsymbol{\rho})$ in isotropic incompressible turbulence (in the absence of inversion symmetry):

$$
F_{2,1}^{\alpha \beta}(\boldsymbol{\rho})=\epsilon_{\alpha \beta \gamma} \hat{\rho}_{\gamma} F_{2,1}(\rho), \quad F_{2,1}(\rho) \propto \rho^{\beta_{1}},
$$

where $\epsilon_{\alpha \beta \gamma}$ isq the fully antisymmetric unit tensor $\left(\epsilon_{123}=\right.$ $\left.-\epsilon_{213}=1\right)$. As we mentioned, in the presence of inversion symmetry $\boldsymbol{F}_{2}(-\boldsymbol{\rho})=\boldsymbol{F}_{2}(\boldsymbol{\rho})$ and all terms which are odd in $\ell$ in (3.2) are zero. On the other hand this is not the case for $\tilde{\boldsymbol{F}}_{2 \ell}(\boldsymbol{\rho})$, which appears in the fusion of two points in a many-point correlation function. Even if the turbulent flow itself has inversion symmetry, the geometry of all the points appearing in (3.7) can lead to non-vanishing odd $\ell$ components of $\tilde{\boldsymbol{F}}_{2 \ell}$. The positions of the points $\boldsymbol{r}_{1}^{\prime}, \boldsymbol{r}_{2}^{\prime} \ldots$ are such that there is no inversion symmetry around the center of the fusing coordinates $\boldsymbol{r}_{1}$ and $\boldsymbol{r}_{2}$ which is $\boldsymbol{r}_{0}=\left(\boldsymbol{r}_{1}+\boldsymbol{r}_{2}\right) / 2$. Therefore $\tilde{\boldsymbol{F}}_{2 \ell}(\boldsymbol{\rho}) \neq 0$ and because of the same constraints it has the same tensor structure as (3.10):

$$
\tilde{F}_{2,1}^{\alpha \beta}(\boldsymbol{\rho})=\epsilon_{\alpha \beta \gamma} \hat{\rho}_{\gamma} \tilde{F}_{2,1}(\rho), \quad \tilde{F}_{2,1}(\rho) \propto \rho^{\beta_{1}} .
$$

The physical origin of this term in the multipole expansion is a local flux of helicity. Even when the average helicity flux is zero, the local value of the flux conditioned on the velocities fixed at certain coordinates may be non zero. 
The exponent $\beta_{2}$ is the leading exponent describing the rate of decay of the effects of anisotropy, and may be computed using perturbation theory, disregarding the nonperturbative effects which are the subject of this paper, see [20,21] and reference therein. The result is $\beta_{2}=4 / 3$, and again one expects this result to be numerically close to the truth. We do not possess presently any numerical estimates for $\beta_{\ell}$ with higher values of $\ell$, and as we said before it is not guaranteed that these exponents are universal. These issues have to be considered independently in the future.

\section{GENERALIZED BALANCE EQUATION}

\section{A. Derivation of generalized balance equation}

The starting point of this analysis is the Navier-Stokes equations for incompressible flows:

$$
\begin{aligned}
\frac{\partial \boldsymbol{u}(\boldsymbol{r}, t)}{\partial t}+\boldsymbol{u}(\boldsymbol{r}, t) \cdot \boldsymbol{\nabla} \boldsymbol{u}(\boldsymbol{r}, t)+\nabla p(\boldsymbol{r}, t) & =\nu \nabla^{2} \boldsymbol{u}(\boldsymbol{r}, t) \\
\boldsymbol{\nabla} \cdot \boldsymbol{u}(\boldsymbol{r}, t) & =0 .
\end{aligned}
$$

In general we need to add a forcing term to these equations. It was shown in [4] that as far as the balance equations are concerned, the effect of the forcing term is felt in the energy containing scales only. For this reason we do not write the forcing explicitly. As usual the gradient of the pressure in (4.1) is eliminated by applying the transverse projection operator $\overleftrightarrow{P}$. The Navier-Stokes equations takes on the form

$$
\frac{\partial \boldsymbol{u}(\boldsymbol{r}, t)}{\partial t}+\stackrel{\leftrightarrow}{P}[\boldsymbol{u}(\mathbf{r}, t) \cdot \nabla] \boldsymbol{u}(\boldsymbol{r}, t)=\nu \nabla^{2} \boldsymbol{u}(\boldsymbol{r}, t)
$$

The application of $\overleftrightarrow{P}$ to any given vector field $\boldsymbol{a}(\boldsymbol{r})$ is non-local, and has the form:

$$
\overleftrightarrow{P} \boldsymbol{a}(\boldsymbol{r})]_{\alpha}=\int d \boldsymbol{r}^{\prime} P_{\alpha \beta}\left(\boldsymbol{r}^{\prime}\right) a_{\beta}\left(\boldsymbol{r}-\boldsymbol{r}^{\prime}\right)
$$

where the kernel $P_{\alpha \beta}(\boldsymbol{r})$ is the following difference:

$$
P_{\alpha \beta}(\boldsymbol{r})=\delta_{\alpha \beta} \delta(\boldsymbol{r})-P_{\alpha \beta}^{\|}(\boldsymbol{r}) \text {. }
$$

Here $P_{\alpha \beta}^{\|}(\boldsymbol{r})$ is the kernel of the longitudinal projector which appears here due to the effect of the pressure term in the Navier-Stokes equation:

$$
P_{\alpha \beta}^{\|}(\boldsymbol{r})=\frac{1}{4 \pi}\left[\frac{\delta_{\alpha \beta}}{r^{3}}-\frac{3 r_{\alpha} r_{\beta}}{r^{5}}\right] .
$$

Given the equation of motion we can take the time derivative of Eq.(1.8). We find

$$
\begin{aligned}
\frac{\partial \boldsymbol{F}_{n}}{\partial t}= & \sum_{j=1}^{n}\left\langle\boldsymbol{w}\left(\boldsymbol{r}_{1}, \boldsymbol{r}_{1}^{\prime}, t\right) \ldots\right. \\
& \left.\ldots \frac{\partial \boldsymbol{w}\left(\boldsymbol{r}_{j}, \boldsymbol{r}_{j}^{\prime}, t\right)}{\partial t} \ldots \boldsymbol{w}\left(\boldsymbol{r}_{n}, \boldsymbol{r}_{n}^{\prime}, t\right)\right\rangle .
\end{aligned}
$$

Substituting Eq.4.2), and considering the stationary state in which $\partial \overline{\boldsymbol{F}}_{n} / \partial t=0$ we find the balance equations

$$
\mathcal{D}_{n}\left(\boldsymbol{r}_{1}, \boldsymbol{r}_{1}^{\prime} ; \ldots \boldsymbol{r}_{n}, \boldsymbol{r}_{n}^{\prime}\right)=\mathcal{J}_{n}\left(\boldsymbol{r}_{1}, \boldsymbol{r}_{1}^{\prime} ; \ldots \boldsymbol{r}_{n}, \boldsymbol{r}_{n}^{\prime}\right)
$$

where the "interaction" term $\mathcal{D}_{n}$ stems from the nonlinear and pressure terms:

$$
\begin{gathered}
\mathcal{D}_{n}\left(\boldsymbol{r}_{1}, \boldsymbol{r}_{1}^{\prime} ; \ldots \boldsymbol{r}_{n}, \boldsymbol{r}_{n}^{\prime}\right)=\sum_{j=1}^{n}\left\langle\boldsymbol{w}\left(\boldsymbol{r}_{1}, \boldsymbol{r}_{1}^{\prime}\right) \ldots\right. \\
\left.\ldots\left[(\stackrel{\leftrightarrow}{P} \boldsymbol{u} \cdot \nabla \boldsymbol{u})_{j}-(\stackrel{\leftrightarrow}{P} \boldsymbol{u} \cdot \nabla \boldsymbol{u})_{j^{\prime}}\right] \ldots \boldsymbol{w}\left(\boldsymbol{r}_{n}, \boldsymbol{r}_{n}^{\prime}\right)\right\rangle,
\end{gathered}
$$

and the "dissipative" term $\mathcal{J}_{n}$ originates from the viscosity term in the Navier Stokes equation:

$$
\begin{aligned}
& \mathcal{J}_{n}\left(\boldsymbol{r}_{1}, \boldsymbol{r}_{1}^{\prime} ; \ldots \boldsymbol{r}_{n}, \boldsymbol{r}_{n}^{\prime}\right)=\nu \sum_{j=1}^{n}\left(\nabla_{j}^{2}+\nabla_{j^{\prime}}^{2}\right)\left\langle\boldsymbol{w}\left(\boldsymbol{r}_{1}, \boldsymbol{r}_{1}^{\prime}\right) \ldots\right. \\
& \left.\ldots \boldsymbol{w}\left(\boldsymbol{r}_{j}, \boldsymbol{r}_{j}^{\prime}\right) \ldots \boldsymbol{w}\left(\boldsymbol{r}_{n}, \boldsymbol{r}_{n}^{\prime}\right)\right\rangle .
\end{aligned}
$$

In writing these equations we used the fact that in the stationary state the time designation is unneeded. We also used the following short-hand notation:

$$
(\stackrel{\leftrightarrow}{P} \boldsymbol{u} \cdot \nabla \boldsymbol{u})_{j}^{\alpha}=\int d \boldsymbol{r} P_{\alpha \beta}\left(\boldsymbol{r}_{j}-\boldsymbol{r}\right) u_{\gamma}(\boldsymbol{r}) \nabla_{\gamma} u_{\beta}(\boldsymbol{r})
$$

and denoted by $\nabla_{j}^{2}$ the Laplacian operator acting on $\boldsymbol{r}_{j}$. Equation (4.8) can be written explicitly in the form

$$
\begin{aligned}
& \mathcal{D}_{n}^{\alpha_{1} \alpha_{2} \ldots \alpha_{n}}\left(\boldsymbol{r}_{1}, \boldsymbol{r}_{1}^{\prime} ; \ldots \boldsymbol{r}_{n}, \boldsymbol{r}_{n}^{\prime}\right)=\sum_{j=1}^{n} \int d \boldsymbol{r} P_{\alpha_{j} \beta}(\boldsymbol{r}) \\
& \times\left\langle w_{\alpha_{1}}\left(\boldsymbol{r}_{1}, \boldsymbol{r}_{1}^{\prime}\right) \ldots L_{\beta}\left(\boldsymbol{r}_{j}, \boldsymbol{r}_{j}^{\prime}, \boldsymbol{r}\right) \ldots w_{\alpha_{n}}\left(\boldsymbol{r}_{n}, \boldsymbol{r}_{n}^{\prime}\right)\right\rangle,
\end{aligned}
$$

where

$$
\begin{aligned}
L_{\beta}\left(\boldsymbol{r}_{j}, \boldsymbol{r}_{j}^{\prime}, \boldsymbol{r}\right) \equiv & {\left[\boldsymbol{u}\left(\boldsymbol{r}_{j}-\boldsymbol{r}\right) \cdot \nabla_{j} u_{\beta}\left(\boldsymbol{r}_{j}-\boldsymbol{r}\right)\right.} \\
& \left.-\boldsymbol{u}\left(\boldsymbol{r}_{j}^{\prime}-\boldsymbol{r}\right) \cdot \nabla_{j}^{\prime} u_{\beta}\left(\boldsymbol{r}_{j}^{\prime}-\boldsymbol{r}\right)\right]
\end{aligned}
$$

Now we begin to analyze the balance equation (4.7, 4.9, 4.11, 4.12. 


\section{B. Galilean invariance of the generalized balance equation}

The balance equation must be Galilean invariant. Equation (4.9) for $\mathcal{J}_{n}$ depends only on velocity differences and clearly is Galilean invariant. This is not so obvious in the case of Eq. (4.11) for $\mathcal{D}_{n}$ because $L_{\beta}$ in (4.12) contains velocities itself. In order to clarify the Galilean invariance of $\mathcal{D}_{n}$ let us express $L_{\beta}$ via velocity differences only. The first step is to subtract from $u_{\beta}\left(\boldsymbol{r}_{j}-\boldsymbol{r}\right)$ under the derivative $\nabla_{j}$ the velocity $u_{\beta}\left(\boldsymbol{r}_{j}^{\prime}-\boldsymbol{r}\right)$ (independent of $\boldsymbol{r}_{j}$ ) and from $u_{\beta}\left(\boldsymbol{r}_{j}^{\prime}-\boldsymbol{r}\right)$ under the derivative $\boldsymbol{\nabla}_{j}^{\prime}$ the velocity $u_{\beta}\left(\boldsymbol{r}_{j}-\boldsymbol{r}\right)$ (independent of $\boldsymbol{r}_{j}^{\prime}$ ). Then Eq. (4.12) takes the form

$$
\begin{aligned}
L_{\beta}\left(\boldsymbol{r}_{j}, \boldsymbol{r}_{j}^{\prime}, \boldsymbol{r}\right)= & {\left[\boldsymbol{u}\left(\boldsymbol{r}_{j}-\boldsymbol{r}\right) \cdot \nabla_{j}+\boldsymbol{u}\left(\boldsymbol{r}_{j}^{\prime}-\boldsymbol{r}\right) \cdot \nabla_{j}^{\prime}\right] } \\
& \times w_{\beta}\left(\boldsymbol{r}_{j}-\boldsymbol{r}, \boldsymbol{r}_{j}^{\prime}-\boldsymbol{r}\right) .
\end{aligned}
$$

For the next step let us introduce $\overline{\boldsymbol{u}}\left(\boldsymbol{r}_{1}, \boldsymbol{r}_{1}^{\prime} ; \ldots ; \boldsymbol{r}_{n}, \boldsymbol{r}_{n}^{\prime}\right)$ as the mean velocity over all $2 n$ space coordinates:

$$
\overline{\boldsymbol{u}} \equiv \frac{1}{2 n} \sum_{k=1}^{n}\left[\boldsymbol{u}\left(\boldsymbol{r}_{k}\right)+\boldsymbol{u}\left(\boldsymbol{r}_{k}^{\prime}\right)\right]
$$

and the following velocity differences:

$$
\boldsymbol{w}\left(\boldsymbol{r}_{j}\right) \equiv \boldsymbol{u}\left(\boldsymbol{r}_{j}\right)-\overline{\boldsymbol{u}}, \quad \boldsymbol{w}\left(\boldsymbol{r}_{j}^{\prime}\right) \equiv \boldsymbol{u}\left(\boldsymbol{r}_{j}^{\prime}\right)-\overline{\boldsymbol{u}} .
$$

For brevity we do not display here (and below) arguments of the velocity $\overline{\boldsymbol{u}}$. Using (4.15) we can present $L_{\beta}$ in 4.12 ) as a sum of two terms:

$$
L_{\beta}\left(\boldsymbol{r}_{j}, \boldsymbol{r}_{j}^{\prime}, \boldsymbol{r}\right)=L_{\beta}^{(1)}\left(\boldsymbol{r}_{j}, \boldsymbol{r}_{j}^{\prime}, \boldsymbol{r}\right)+L_{\beta}^{(2)}\left(\boldsymbol{r}_{j}, \boldsymbol{r}_{j}^{\prime}, \boldsymbol{r}\right) .
$$

Here the first term depends only on velocity differences:

$$
\begin{aligned}
L_{\beta}^{(1)}\left(\boldsymbol{r}_{j}, \boldsymbol{r}_{j}^{\prime}, \boldsymbol{r}\right)= & {\left[\boldsymbol{w}\left(\boldsymbol{r}_{j}-\boldsymbol{r}\right) \cdot \nabla_{j}\right.} \\
& \left.+\boldsymbol{w}\left(\boldsymbol{r}_{j}^{\prime}-\boldsymbol{r}\right) \cdot \nabla_{j}^{\prime}\right] w_{\beta}\left(\boldsymbol{r}_{j}-\boldsymbol{r}, \boldsymbol{r}_{j}^{\prime}-\boldsymbol{r}\right) .
\end{aligned}
$$

However the second term does not have this property:

$$
L_{\beta}^{(2)}\left(\boldsymbol{r}_{j}, \boldsymbol{r}_{j}^{\prime}, \boldsymbol{r}\right)=\overline{\boldsymbol{u}} \cdot\left[\boldsymbol{\nabla}_{j}+\cdot \nabla_{j}^{\prime}\right] w_{\beta}\left(\boldsymbol{r}_{j}-\boldsymbol{r}, \boldsymbol{r}_{j}^{\prime}-\boldsymbol{r}\right)
$$

but, as we are going to show, this term gives zero contribution to Eq. (4.11) for $\mathcal{D}_{n}$. Indeed, by substituting (4.18) in (4.11) we have:

$$
\begin{gathered}
\mathcal{D}_{n}^{(2)}=\sum_{j=1}^{n} \int d \boldsymbol{r}_{j} P_{\alpha_{j} \beta}(\boldsymbol{r}) \\
\times\left\langle w_{\alpha_{1}}\left(\boldsymbol{r}_{1}, \boldsymbol{r}_{1}^{\prime}\right) \ldots L_{2}^{\beta}\left(\boldsymbol{r}_{j}, \boldsymbol{r}_{j}^{\prime}, \boldsymbol{r}\right) \ldots w_{\alpha_{n}}\left(\boldsymbol{r}_{n}, \boldsymbol{r}_{n}^{\prime}\right)\right\rangle .
\end{gathered}
$$

In its turn, this equation may be written as the difference of two terms, $\mathcal{D}_{n}^{(2)}=\mathcal{D}_{n}^{(2 a)}-\mathcal{D}_{n}^{(2 b)}$, which correspond to the two terms in the Eq. 4.4) for the kernel $P_{\alpha_{j} \beta}(\boldsymbol{r})$. By substituting in (4.19) $P_{\alpha \beta}(\boldsymbol{r})=\delta_{\alpha \beta} \delta(\boldsymbol{r})$ we have:
$\mathcal{D}_{n}^{(2 a)}=\sum_{j=1}^{n}\left\langle w_{\alpha_{1}}\left(\boldsymbol{r}_{1}, \boldsymbol{r}_{1}^{\prime}\right) \ldots L_{2}^{\beta}\left(\boldsymbol{r}_{j}, \boldsymbol{r}_{j}^{\prime}, 0\right) \ldots w_{\alpha_{n}}\left(\boldsymbol{r}_{n}, \boldsymbol{r}_{n}^{\prime}\right)\right\rangle$

Using the longitudinal projector $P_{\alpha_{j} \beta}^{\|}(\boldsymbol{r})$ instead of the transversal one $P_{\alpha_{j} \beta}(\boldsymbol{r})$ in Eq. (4.19) we have:

$$
\begin{aligned}
\mathcal{D}_{n}^{(2 b)} & =\sum_{j=1}^{n} \int d \boldsymbol{r}_{j} P_{\alpha_{j} \beta}^{\|}(\boldsymbol{r}) \\
& \times\left\langle w_{\alpha_{1}}\left(\boldsymbol{r}_{1}, \boldsymbol{r}_{1}^{\prime}\right) \ldots L_{\beta}^{(2)}\left(\boldsymbol{r}_{j}, \boldsymbol{r}_{j}^{\prime}, \boldsymbol{r}\right) \ldots w_{\alpha_{n}}\left(\boldsymbol{r}_{n}, \boldsymbol{r}_{n}^{\prime}\right)\right\rangle .
\end{aligned}
$$

Let us show that both these terms are zero (but because of different reasons). Consider the first expression for $\mathcal{D}_{n}^{(2 a)}$. Substituting the explicit form (4.18) for $L_{\beta}^{(2)}\left(\boldsymbol{r}_{j}, \boldsymbol{r}_{j}^{\prime}, 0\right)$ and using the incompressibility constraint (which allows one to commute $\boldsymbol{\nabla}_{j}+\boldsymbol{\nabla}_{j}^{\prime}$ and $\overline{\boldsymbol{u}}$ ) one has:

$$
\begin{aligned}
& \mathcal{D}_{n}^{(2 a)}=\sum_{j=1}^{n}\left\langle w_{\alpha_{1}}\left(\boldsymbol{r}_{1}, \boldsymbol{r}_{1}^{\prime}\right)\right. \\
& \left.\ldots\left\{\left[\left(\boldsymbol{\nabla}_{j}+\nabla_{j}^{\prime}\right) \cdot \overline{\boldsymbol{u}}\right] w_{\alpha_{j}}\left(\boldsymbol{r}_{j}, \boldsymbol{r}_{j}^{\prime}\right)\right\} \ldots w_{\alpha_{n}}\left(\boldsymbol{r}_{n}, \boldsymbol{r}_{n}^{\prime}\right)\right\rangle .
\end{aligned}
$$

This equation may be rewritten as

$$
\begin{gathered}
\mathcal{D}_{n}^{(2 a)}=\sum_{j=1}^{n}\left(\nabla_{j}^{\beta}+\nabla_{j}^{\prime \beta}\right) \\
\times\left\langle\bar{u}^{\beta} w_{\alpha_{1}}\left(\boldsymbol{r}_{1}, \boldsymbol{r}_{1}^{\prime}\right) w_{\alpha_{2}}\left(\boldsymbol{r}_{j}, \boldsymbol{r}_{2}^{\prime}\right) \ldots w_{\alpha_{n}}\left(\boldsymbol{r}_{n}, \boldsymbol{r}_{n}^{\prime}\right)\right\rangle .
\end{gathered}
$$

Remember that due to space homogeneity the correlation function in the second line of this equation is independent of the sum of coordinates $\sum_{j=1}^{n}\left(\boldsymbol{r}_{j}+\boldsymbol{r}_{j}^{\prime}\right)$. Therefore $\mathcal{D}_{n}^{(2 a)}$ is indeed equal to zero.

Consider next Eq. (4.21) for $\mathcal{D}_{n}^{(2 b)}$. According to (4.18) $L_{\beta}^{(2)} \propto w_{\beta}\left(\boldsymbol{r}_{j}-\boldsymbol{r}, \boldsymbol{r}_{j}^{\prime}-\boldsymbol{r}\right)$ and acting on this velocity with the longitudinal projector gives zero because of the incompressibility constraint. Thus we can conclude that $\mathcal{D}_{n}^{(2 a)}=\mathcal{D}_{n}^{(2 b)}=0$. Therefore we can get an expression for $\mathcal{D}_{n}$ by replacing $L_{\beta}$ in Eq. (4.11) with $L_{\beta}^{(1)}$ taken from Eq. (4.17):

$$
\begin{aligned}
& \mathcal{D}_{n}^{\alpha_{1} \alpha_{2} \ldots \alpha_{n}}\left(\boldsymbol{r}_{1}, \boldsymbol{r}_{1}^{\prime} ; \ldots \boldsymbol{r}_{n}, \boldsymbol{r}_{n}^{\prime}\right)=-\int d \boldsymbol{r} \sum_{j=1}^{n} P_{\alpha_{j} \beta}(\boldsymbol{r}) \\
\times & \left\langlew _ { \alpha _ { 1 } } ( \boldsymbol { r } _ { 1 } , \boldsymbol { r } _ { 1 } ^ { \prime } ) \ldots \left\{\left[u_{\gamma}\left(\boldsymbol{r}_{j}-\boldsymbol{r}\right)-\bar{u}_{\gamma}\right] \frac{\partial}{\partial r_{j \gamma}}\right.\right. \\
+ & {\left.\left[u_{\gamma}\left(\boldsymbol{r}_{j}^{\prime}-\boldsymbol{r}\right)-\bar{u}_{\gamma}\right] \frac{\partial}{\partial r_{j \gamma}^{\prime}}\right\} } \\
\times & \left.w_{\beta}\left(\boldsymbol{r}_{j}-\boldsymbol{r}, \boldsymbol{r}_{j}^{\prime}-\boldsymbol{r}\right) \ldots w_{\alpha_{n}}\left(\boldsymbol{r}_{n}, \boldsymbol{r}_{n}^{\prime}\right)\right\rangle .
\end{aligned}
$$

This expression for $\mathcal{D}_{n}$ depends only on velocity differences and therefore the Galilean invariance becomes obvious. 


\section{Locality of the Interaction Term}

We begin to analyze the interaction terms $\mathcal{D}_{n}(4.24)$ for the most general configuration for which all the $2 n$ coordinates $\boldsymbol{r}_{j}, \boldsymbol{r}_{j}^{\prime}$ are different, and all the $n(2 n-1)$ separations are of the same order of magnitude, which we designate by $R$. This analysis when $R$ is in the inertial interval follows the ideas of the analysis presented in (4) section $6 \mathrm{~A}$ for the interaction term of the structure function. The interaction term that we face here is a significantly more complicated object. The main point is that the integral over $\boldsymbol{r}$ appearing in Eq. (4.24) for $\mathcal{D}_{n}$ is "local" in the following sense. First it converges in the "ultra-violet" (UV) limit. This limit has to be considered when (i) $r \rightarrow 0$, (ii) when $\left(\boldsymbol{r}_{j}-\boldsymbol{r}\right)$ becomes very close to any of the $2 n-1$ coordinates other than $r_{j}$, and (iii) when $\left(\boldsymbol{r}_{j}^{\prime}-\boldsymbol{r}\right)$ becomes very close to any of the $2 n-1$ coordinates other than $r_{j}^{\prime}$. Second, it converges in the "infra-red" (IR) limit when $r \rightarrow \infty$. The idea for the proof of these properties lies in the use of the fusion rules which we discussed in Sect. II.

\section{Ultraviolet convergence}

To demonstrate the convergence of the integral in $\mathcal{D}_{n}$ in the ultraviolet region we can consider any term from the sum on $j$. Writing $u_{\gamma}\left(\boldsymbol{r}_{j}-\boldsymbol{r}\right)=\left(\sum_{k=1}^{n} u_{\gamma}\left(\boldsymbol{r}_{j}-\boldsymbol{r}\right)\right) / n$, and using Eq.(4.14), we consider one of the $k$-terms in the sum. The integral that appears is of the form

$$
\begin{aligned}
& I=\frac{1}{n} \int d \boldsymbol{r} P_{\alpha_{j} \beta}(\boldsymbol{r}) \frac{\partial}{\partial r_{j \gamma}}\left\langle w_{\alpha_{1}}\left(\boldsymbol{r}_{1}, \boldsymbol{r}_{1}^{\prime}\right) \ldots\right. \\
& \left.w_{\gamma}\left(\boldsymbol{r}_{j}-\boldsymbol{r}, \boldsymbol{r}_{k}\right) w_{\beta}\left(\boldsymbol{r}_{j}-\boldsymbol{r}, \boldsymbol{r}_{j}^{\prime}-\boldsymbol{r}\right) \ldots w_{\alpha_{n}}\left(\boldsymbol{r}_{n}, \boldsymbol{r}_{n}^{\prime}\right)\right\rangle .
\end{aligned}
$$

As the coordinate $\boldsymbol{r}$ is being integrated over, the most dangerous ultraviolet contribution comes from the region of small $r$. In this region the projection operator can be evaluated as $1 / r^{3}$. Other coalescence events of $\boldsymbol{r}$ with other coordinates contribute less divergent integrands since the projection operator does not become singular. When $r$ becomes small, there are two possibilities: (i) $\boldsymbol{r}_{j} \neq \boldsymbol{r}_{k}$ and (ii) $\boldsymbol{r}_{j}=\boldsymbol{r}_{k}$. In the first case the correlation function itself is analytic in the region $r \rightarrow 0$, and we can expand it in a Taylor series Const $+\boldsymbol{B} \cdot \boldsymbol{r}+\ldots$. where $\boldsymbol{B}$ is an $\boldsymbol{r}$-independent vector. The constant term is annihilated by the projection operator. The term linear in $\boldsymbol{r}$ vanishes under the $d \boldsymbol{r}$ integration due to $\boldsymbol{r} \rightarrow-\boldsymbol{r}$ symmetry. The next term which is proportional to $r^{2}$ is convergent in the ultraviolet. In the second case we have a velocity difference across the length $r$. Accordingly we need to use the fusion rule (A4), and we learn that the leading contribution is proportional to $r^{\zeta_{2}}$. This is sufficient for convergence in the ultraviolet. We note that the derivative with respect to $r_{j}$ cannot be evaluated as $1 / r$ when $\boldsymbol{r}_{j}=\boldsymbol{r}_{k}$. Rather, it is evaluated as the inverse of the distance between $\boldsymbol{r}_{j}$ and the nearest coordinate in the correlation function.

\section{Infrared convergence}

FIG. IV C 2, Typical geometry with $(n-1)$ velocity differences in a ball of radius $R$ on the left separated by a large distance $r \gg R$ from a pair of points on the right.

To understand the convergence of $\mathcal{D}_{n}$ when the integration variable $r$ becomes very large we can consider again the typical term (4.25). The relevant geometry is shown in Fig. IV C2 . There is one velocity difference across the coordinates $\boldsymbol{r}_{j}-\boldsymbol{r}$ and $\boldsymbol{r}_{j}^{\prime}-\boldsymbol{r}$ (which is shown on the right of the figure), $(n-1)$ velocity differences across coordinates that are all within a ball of radius $R$ (at the left of the figure), and one velocity difference across the large distance $r$ which is much larger than $R$. In the notation of this figure the leading order contribution for large $r$ is obtained from the fusion rules (A4) for the situation on the right and (2.8) for the geometry on the left. The resulting evaluation for the leading term is

$$
I \propto r^{\zeta_{n+1}}\left(\frac{R_{j}}{r}\right)^{\zeta_{2}}\left(\frac{R}{r}\right)^{\zeta_{n-1}} .
$$

On the face of it, this term is near dangerous. For K41 scaling the $r$-dependence cancels, and the integral is logarithmically divergent. For anomalous scaling the integral converges since $\zeta_{n+1} \leq \zeta_{n-1}+\zeta_{2}$ due to Hoelder inequalities. This convergence seems slow. However, the situation is in fact much safer. If we take into account the precise form of the second-order structure function in the fusion rules we find that the divergence with respect to $\boldsymbol{r}_{j}$ translates in fact to $\partial S_{2}^{\beta \gamma}\left(\boldsymbol{R}_{j}\right) / \partial R_{j \gamma}$ which is zero due to incompressibility. The next order term is convergent even for simple (K41) scaling. This completes the proof of locality of (4.8). The conclusion is that when all the separations in $\boldsymbol{D}_{n}$ are of the same order of magnitude $R$, the main contribution to the integral in (4.8) comes from the region $r \sim R$. Therefore, the integral can be evaluated by straightforward power counting, leading to

$$
\mathcal{D}_{n} \sim \frac{S_{n+1}(R)}{R}
$$

It should be stressed that a more detailed analysis demonstrates that when the separations $\boldsymbol{\rho}$ between the coordinates that do not involve velocity differences, (i.e separations like $\boldsymbol{r}_{j k}$ but not $\boldsymbol{R}_{j}$ ) go to zero, the evaluation does not change. A direct proof of this fact for the case when all such separations are fused (i.e the standard structure function) was presented in 任. On the other hand, if we consider fusions of type $\mathrm{A}$, in which coordinates across a velocity difference coalesce $\left(\boldsymbol{\rho}=\boldsymbol{R}_{j}\right)$, we need to be more careful. When two points 
undergo a fusion of type A the rough evaluation of $\mathcal{D}_{n}$ is $\left[\left(d S_{2}(\rho) / d \rho\right)\right]\left[S_{n+1}(R) / S_{2}(R)\right]$ where $R$ is the characteristic value of large separations. However taking into account the tensor structure of the first factor one sees that it vanishes due to the incompressibility constraint. In the next-order term the evaluation of the gradient is $1 / R$ and $\mathcal{D}_{n} \rightarrow 0$ when $\rho \rightarrow 0$.

\section{The dissipative term}

The evaluation of the quantity $\mathcal{J}_{n}$ is more straightforward. When all the separations $R_{j}$ and $r_{i j}$ are of the same order $R$, the correlator in (4.9) is evaluated simply as $S_{n}(R)$. The Laplacian is then of the order of $1 / R^{2}$. The evaluation is

$$
\mathcal{J}_{n} \sim \nu \frac{S_{n}(R)}{R^{2}} .
$$

When one of the separations becomes much smaller than the rest the evaluation can be read directly from the definition (59) and from the fusion rules. Denoting the smallest separation by $r_{\min }$ we write

$$
\mathcal{J}_{n} \sim \nu S_{2}\left(r_{\min }\right) \frac{S_{n}(R)}{S_{2}(R) r_{\text {min }}^{2}} .
$$

\section{THE HOMOGENEOUS EQUATION}

We noted that when all the separations involved in our correlation functions are of the same order of magnitude, and when $\nu \rightarrow 0$ (which is the limit of infinite Reynolds number Re), the term $\mathcal{J}_{n}$ becomes negligible compared to $\mathcal{D}_{n}$. The ratio $\mathcal{J}_{n} / \mathcal{D}_{n}$ is evaluated as $\nu S_{n}(R) / R S_{n+1}(R)$, which for fixed $R$ vanishes in the limit $\nu \rightarrow 0$. Thus the "balance equation" becomes a homogeneous integro-differential equation $\mathcal{D}_{n}=0$ which may have scale-invariant solutions with anomalous scaling exponents $\zeta_{n+1} \neq(n+1) / 3$. It should be stressed that the evaluation (4.27) remains correct for every term in $\mathcal{D}_{n}$, but various terms cancel to give zero in the homogeneous equation, provided that the scaling exponent $\zeta_{n}$ is chosen correctly. To make this important point clear we exemplify it with the simple case $n=2$ for which $\mathcal{D}_{n}$ can be greatly simplified. Consider the scalar object $\mathcal{F}_{2}\left(\boldsymbol{r}_{1}, \boldsymbol{r}_{1}^{\prime}, \boldsymbol{r}_{2}, \boldsymbol{r}_{2}^{\prime}\right)=\left\langle\boldsymbol{w}\left(\boldsymbol{r}_{1} \mid \boldsymbol{r}_{1}^{\prime}\right) \cdot \boldsymbol{w}\left(\boldsymbol{r}_{2} \mid \boldsymbol{r}_{2}^{\prime}\right)\right\rangle$. The terms in the scalar balance equation for this case are exactly

$$
\begin{aligned}
\mathcal{D}_{2}\left(\boldsymbol{r}_{1}, \boldsymbol{r}_{1}^{\prime}, \boldsymbol{r}_{2}, \boldsymbol{r}_{2}^{\prime}\right) & =d\left[S_{3}\left(r_{12^{\prime}}\right)-S_{3}\left(r_{12}\right)\right] / 2 d r_{1} \\
& +d\left[S_{3}\left(r_{1^{\prime} 2}\right)-S_{3}\left(r_{1^{\prime} 2^{\prime}}\right)\right] / 2 d r_{1}^{\prime}, \\
\mathcal{J}_{2}\left(\boldsymbol{r}_{1}, \boldsymbol{r}_{1}^{\prime}, \boldsymbol{r}_{2}, \boldsymbol{r}_{2}^{\prime}\right) & =\nu\left\{\nabla_{1}^{2}\left[S_{2}\left(r_{12^{\prime}}\right)-S_{2}\left(r_{12}\right)\right]\right. \\
& \left.+\nabla_{1^{\prime}}^{2}\left[S_{2}\left(r_{1^{\prime} 2}\right)-S_{2}\left(r_{1^{\prime} 2^{\prime}}\right)\right]\right\}
\end{aligned}
$$

When all the separations are of the order of $R$ we can see explicitly that $\mathcal{J}_{2} \sim \nu S_{2}(R) / R^{2}$ which is much smaller than each term in $\mathcal{D}_{2}$. Considering the scale invariant solution $S_{3}(R)=A R^{\zeta_{3}}$ where $A$ is a dimensional coefficient, we see that

$\mathcal{D}_{2}\left(\boldsymbol{r}_{1}, \boldsymbol{r}_{1}^{\prime}, \boldsymbol{r}_{2}, \boldsymbol{r}_{2}^{\prime}\right)=\frac{\zeta_{3} A}{2}\left[r_{12^{\prime}}^{\zeta_{3}-1}-r_{12}^{\zeta_{3}-1}+r_{1^{\prime} 2}^{\zeta_{3}-1}-r_{1^{\prime} 2^{\prime}}^{\zeta_{3}-1}\right]$.

Obviously the solution for $\mathcal{D}_{2}=0$ requires the unique choice $\zeta_{3}=1$ which is the known exponent for $S_{3}$ [14]. The coefficient $A$ is now determined as $\bar{\epsilon}$ which is the mean energy dissipation per unit mass and unit time.

We presently do not know how to find the homogeneous solution of the equation $\mathcal{D}_{n}=0$ for higher values of $n$. We are even not fully confident that this is an equation in the usual sense and not just a constraint that will not be sufficient for a unique determination of the scaling exponents $\zeta_{n}$. We feel however that this is an interesting equation that will offer interesting and worthwhile insights.

\section{THE DISSIPATIVE SCALING FUNCTIONS}

In this section we consider the dissipative "scales" and show that they are actually scaling functions. To define properly the dissipative length we use the fact that there is a cross-over from the scale invariant solution of the homogeneous equation to dissipative solutions when $\mathcal{J}_{2}$ becomes comparable to any of the terms in $\mathcal{D}_{2}$. This happens when at least one of the separations appearing in (5.2) becomes small enough. Denoting the smallest separation as $r_{\min }$ we evaluate $\mathcal{J}_{2} \sim \nu S_{2}\left(r_{\min }\right) / r_{\min }^{2}$. From this we can estimate, using the balance equation, $S_{2}\left(r_{\text {min }}\right) \sim\left(S_{3}(R) / \nu R\right) r_{\text {min }}^{2} \sim \bar{\epsilon} r_{\text {min }}^{2} / \nu$. In the inertial range we have $S_{2}(r) \sim(\bar{\epsilon} r)^{2 / 3}(r / L)^{\zeta_{2}-2 / 3}$. The viscous scale $\eta_{2}$ for the second-order structure function is then determined from finding where these two expressions are of the same order of magnitude, i.e.

$$
\bar{\epsilon} \eta_{2}^{2} / \nu=\left(\bar{\epsilon} \eta_{2}\right)^{2 / 3}(r / L)^{\zeta_{2}-2 / 3}
$$

Using the outer velocity scale $U_{L}$ we estimate $\bar{\epsilon} \sim U_{L}^{3} / L$ and end up with

$$
\eta_{2} \sim L \mathrm{Re}^{-1 /\left(2-\zeta_{2}\right)}
$$

Note that this result is not in agreement with the adhoc application of the multifractal model [14,22,23] which predicts $\eta_{2} \sim L \mathrm{Re}^{-2 /\left(2+\zeta_{2}\right)}$.

A similar mechanism operates in the general case of $n \neq 2$. As long as all the separations are in the inertial interval $\mathcal{J}_{n}$ is negligible. When one separation e.g. $r_{12}$ diminishes towards zero, and all the other separations are of the order of $R$, the internal cancellations leading to the homogeneous equation $\mathcal{D}_{n}=0$ disappear, and $\mathcal{D}_{n}$ is evaluated as in (4.27). The term $\mathcal{J}_{n}$ is now dominated by one contribution that can be written in short-hand notation as $\nu \nabla_{1}^{2} F_{n}\left(r_{12},\{R\}\right)$. We can solve for $F_{n}\left(r_{12},\{R\}\right)$ in this limit: 


$$
F_{n}\left(r_{12},\{R\}\right) \approx r_{12}^{2} S_{n+1}(R) / \nu R .
$$

On the other hand we have, from the fusion rule (2.28), the form of the same quantity when $r_{12}$ is still in the inertial range, i.e. $F_{n}\left(r_{12},\{R\}\right) \approx S_{2}\left(r_{12}\right) S_{n}(R) / S_{2}(R)$. To estimate the viscous scale $\eta_{n}$ we find when these two evaluations are of the same order. The answer is

$$
\eta_{n}(R)=\eta_{2}\left(\frac{R}{L}\right)^{x_{n}}, \quad x_{n}=\frac{\zeta_{n}+\zeta_{3}-\zeta_{n+1}-\zeta_{2}}{2-\zeta_{2}} .
$$

We note that the Hoelder inequalities guarantee that $x_{n}>0$ and increases with $n$. We see that the viscous "length" is actually an anomalous scaling function.

\section{EXACT BRIDGE RELATIONS}

In this section we derive important (and exact) scaling relations between the exponents $\zeta_{n}$ of the structure functions and exponents involving correlations of the dissipation field. We consider correlations of the type

$$
\begin{aligned}
\mathcal{K}_{\epsilon}^{(n)} & \equiv\left\langle\epsilon\left(\boldsymbol{x}_{1}\right) \boldsymbol{w}\left(\boldsymbol{r}_{1} \mid \boldsymbol{r}_{1}^{\prime}\right) \ldots \boldsymbol{w}\left(\boldsymbol{r}_{n} \mid \boldsymbol{r}_{n}^{\prime}\right)\right\rangle \propto R^{-\mu_{n}^{(1)}} \\
\mathcal{K}_{2 \epsilon}^{(n)} & \equiv\left\langle\epsilon\left(\boldsymbol{x}_{1}\right) \epsilon\left(\boldsymbol{x}_{2}\right) \boldsymbol{w}\left(\boldsymbol{r}_{1} \mid \boldsymbol{r}_{1}^{\prime}\right) \ldots \boldsymbol{w}\left(\boldsymbol{r}_{n} \mid \boldsymbol{r}_{n}^{\prime}\right)\right\rangle \propto R^{-\mu_{n}^{(2)}} \\
\mathcal{K}_{p \epsilon}^{(n)} & \equiv\left\langle\epsilon\left(\boldsymbol{x}_{1}\right) \epsilon\left(\boldsymbol{x}_{2}\right) \ldots \epsilon\left(\boldsymbol{x}_{p}\right) \boldsymbol{w}\left(\boldsymbol{r}_{1} \mid \boldsymbol{r}_{1}^{\prime}\right) \ldots \boldsymbol{w}\left(\boldsymbol{r}_{n} \mid \boldsymbol{r}_{n}^{\prime}\right)\right\rangle \\
& \propto R^{-\mu_{n}^{(p)}}
\end{aligned}
$$

where $R$ is a typical separation between any pair and $\epsilon(\boldsymbol{x}) \equiv \nu|\nabla \boldsymbol{u}(\boldsymbol{x})|^{2}$, and we are interested in the scaling relations between the exponents $\mu_{n}^{(p)}$ and the exponents $\zeta_{n}$. Note that $\mu_{0}^{(2)}$ in this notation is the well studied 17, 12 exponent of dissipation fluctuation which is denoted $\mu$. We begin with the rigorous calculation of $\mu_{n}^{(1)}$.

Consider (4.9) for $\mathcal{J}_{n+2}\left(\boldsymbol{x}_{1}, \boldsymbol{x}_{1}^{\prime} ; \boldsymbol{x}_{2}, \boldsymbol{x}_{2}^{\prime} ; \boldsymbol{r}_{1}, \boldsymbol{r}_{1}^{\prime} \ldots \boldsymbol{r}_{n}, \boldsymbol{r}_{n}^{\prime}\right)$ in the limit $\boldsymbol{x}_{1} \rightarrow \boldsymbol{x}_{2}$. The leading contribution in the limit arises from the Laplacians with respect to the coalescing points:

$$
\begin{aligned}
\lim _{x_{1} \rightarrow x_{2}} \mathcal{J}_{n+2} & =\nu \lim _{x_{1} \rightarrow x_{2}}\left(\nabla_{1}^{2}+\nabla_{2}^{2}\right)\left\langle\boldsymbol{u}\left(\boldsymbol{x}_{1}\right) \boldsymbol{u}\left(\boldsymbol{x}_{2}\right)\right. \\
& \left.\times \boldsymbol{w}\left(\boldsymbol{r}_{1}, \boldsymbol{r}_{1}^{\prime}\right) \ldots \boldsymbol{w}\left(\boldsymbol{r}_{n}, \boldsymbol{r}_{n}^{\prime}\right)\right\rangle .
\end{aligned}
$$

Moving one gradient around and taking the trace with respect to the first two tensor indices we see that in this limit

$$
\lim _{r_{1} \rightarrow r_{2}} \mathcal{J}_{n+2}^{\alpha \alpha}=-2 \mathcal{K}_{\epsilon}^{(n)} .
$$

As explained in the previous section, when the two points coalesce $\mathcal{D}_{n+2}$ of the balance equation loses its internal cancellations, and we can therefore conclude immediately that

$$
\mathcal{K}_{\epsilon}^{(n)} \sim \frac{S_{n+3}(R)}{R} .
$$

In terms of the scaling exponents we are led to the exact relation

$$
\mu_{n}^{(1)}=1-\zeta_{n+3} .
$$

The scaling relations satisfied by $\mu_{n}^{(2)}$ require considerations of the second time derivative of the correlation (1.8).

$$
\begin{aligned}
\ddot{\mathcal{F}}_{n}= & \sum_{i, j=1}^{n}\left\langle\boldsymbol{w}\left(\boldsymbol{r}_{1} \mid \boldsymbol{r}_{1}^{\prime}, t\right) \ldots \dot{\boldsymbol{w}}\left(\boldsymbol{r}_{i} \mid \boldsymbol{r}_{i}^{\prime}, t\right)\right. \\
& \left.\ldots \dot{\boldsymbol{w}}\left(\boldsymbol{r}_{j} \mid \boldsymbol{r}_{j}^{\prime}, t\right) \ldots \boldsymbol{w}\left(\boldsymbol{r}_{n} \mid \boldsymbol{r}_{n}^{\prime}, t\right)\right\rangle .
\end{aligned}
$$

Using the Navier-Stokes equations for the time derivatives we derive a new balance equation $\mathcal{D}_{n}^{(2)}+\boldsymbol{B}_{n}^{(2)}=\mathcal{J}_{n}^{(2)}$ where, using the definition (4.12),

$$
\begin{aligned}
& \mathcal{D}_{n}^{(2)}=\int d \boldsymbol{r} d \boldsymbol{r}^{\prime} \sum_{i, j=1}^{n} \boldsymbol{P}(\boldsymbol{r}) \boldsymbol{P}\left(\boldsymbol{r}^{\prime}\right)\left\langle\boldsymbol{w}\left(\boldsymbol{r}_{1} \mid \boldsymbol{r}_{1}^{\prime}\right)\right. \\
& \left.\ldots \boldsymbol{L}\left(\boldsymbol{r}_{i}, \boldsymbol{r}_{i}^{\prime}, \boldsymbol{r}\right) \ldots \boldsymbol{L}\left(\boldsymbol{r}_{j}, \boldsymbol{r}_{j}^{\prime}, \boldsymbol{r}^{\prime}\right) \ldots \boldsymbol{w}\left(\boldsymbol{r}_{n} \mid \boldsymbol{r}_{n}^{\prime}\right)\right\rangle .
\end{aligned}
$$

Using the fusion rules and following steps similar to those described above, we can prove that the integrals over $\boldsymbol{r}$ and $\boldsymbol{r}^{\prime}$ converge. Accordingly, when all the separations are of the order of $R$, every term in $\mathcal{D}_{n}^{(2)}$ is evaluated as $S_{n+2}(R) / R^{2}$. The term $\mathcal{J}_{n}^{(2)}$ takes on the form

$$
\begin{gathered}
\mathcal{J}_{n}^{(2)}=\nu^{2} \sum_{i, j=1}^{n}\left(\nabla_{i}^{2}+\nabla_{i^{\prime}}^{2}\right)\left(\nabla_{j}^{2}+\nabla_{j^{\prime}}^{2}\right) \\
\times\left\langle\boldsymbol{w}\left(\boldsymbol{r}_{1} \mid \boldsymbol{r}_{1}^{\prime}\right) \ldots \boldsymbol{w}\left(\boldsymbol{r}_{i} \mid \boldsymbol{r}_{i}^{\prime}\right) \ldots \boldsymbol{w}\left(\boldsymbol{r}_{j} \mid \boldsymbol{r}_{j}^{\prime}\right) \ldots \boldsymbol{w}\left(\boldsymbol{r}_{n} \mid \boldsymbol{r}_{n}^{\prime}\right)\right\rangle .
\end{gathered}
$$

As before, when all the separation in this quantity are of the order of $R$, the Laplacian operators introduce factor of $1 / R^{2}$ and the evaluation of this quantity is $\mathcal{J}_{n}^{(2)} \sim \nu^{2} S_{n}(R) / R^{4}$. Clearly this is negligible compared to typical terms in $\mathcal{D}_{n}^{(2)}$. The quantity $\boldsymbol{B}_{n}^{(2)}$ contains a cross contribution with one Laplacian operator and one nonlinear term with a projection operator. The integral is again local, and one can show that the evaluation is $\mathcal{B}_{n}^{(2)} \sim \nu S_{n+1}(R) / R^{3}$ which is also negligible compared to typical terms in $\mathcal{D}_{n}^{(2)}$.

Now we consider the fusion of two pairs of coordinate, e.g. $r_{12} \rightarrow 0$ and $r_{34} \rightarrow 0$. As before, the cancellations in $\mathcal{D}_{n}^{(2)}$ are eliminated, and the evaluation of a typical term becomes the evaluation of the quantity. The other two terms in the balance equation also become of the same order because the Laplacian operators $\nabla_{1}^{2}$ and $\nabla_{3}^{2}$ are evaluated as $r_{12}^{-2}$ and $r_{34}^{-2}$ respectively. As before we can consider the resulting balance equation as a differential equation for $F_{n}\left(r_{12}, r_{34},\{R\}\right)$. The leading term in this equation is

$$
4 \nu^{2} \nabla_{1}^{2} \nabla_{2}^{2} F_{n}\left(r_{12}, r_{34},\{R\}\right) \approx \mathcal{B}_{n}^{(2)}+\mathcal{D}_{n}^{(2)} \sim S_{n+2}(R) / R^{2} .
$$

The solution is 


$$
\mathcal{F}_{n}\left(r_{12}, r_{34},\{R\}\right) \sim r_{12}^{2} r_{34}^{2} S_{n+2}(R) / \nu^{2} R^{2} .
$$

Finally we can write the quantities $\mathcal{K}_{\epsilon \epsilon}^{(n)}$ in terms of the correlation function as

$$
\mathcal{K}_{2 \epsilon}^{(n)}=\nu^{2} \lim _{r_{12}, r_{34} \rightarrow 0} \nabla_{1} \nabla_{2} \nabla_{3} \boldsymbol{\nabla}_{4} \mathcal{F}_{n+4}\left(r_{12}, r_{34},\{R\}\right) .
$$

Using (7.11) here we end up with the evaluation

$$
\mathcal{K}_{2 \epsilon}^{(n)} \sim S_{n+6} / R^{2} \propto R^{-\mu_{n}^{(2)}}, \quad \mu_{n}^{(2)}=2-\zeta_{n+6} .
$$

For the standard exponent $\mu=\mu_{0}^{(2)}$ we choose $n=0$ and obtain the phenomenologically proposed "bridge relation"

$$
\mu=2-\zeta_{6} .
$$

To our best knowledge this is the first solid derivation of this scaling relation. In general, if we have $p$ dissipation fields correlated with $n$ velocity differences the scaling exponent can be found by considering $p$ time derivatives of (1.8), with the final result

$$
\mu_{n}^{(p)}=p-\zeta_{n+3 p} .
$$

We see that Eqs.(7.7), (7.13) and (7.15) can be guessed if we assert that for the sake of scaling purposes the dissipation field $\epsilon(\boldsymbol{r})$ can be swapped in the correlation function with $w^{3}\left(\boldsymbol{r}_{1} \mid \boldsymbol{r}_{1}^{\prime}\right) / R_{1}$, where $R_{1}$ is the characteristic scale. This reminds one of the Kolmogorov refined similarity hypothesis. We should stress that (i) our result does not depend on any uncontrolled hypothesis, and (ii) it does not imply the correctness of the hypothesis. Our result is implied by the refined similarity hypothesis, but not vice versa.

We end this section by noting that the accepted values of $\mu$ and $\zeta_{6}$ which are about 0.2 and 1.8 respectively, are in good agreement with the standard bridge relation (7.14). However, we have presented here a "two-dimensional" array of bridge relations depending on the indices $n$ and $p$ whose experimental test with high Reynolds number flows with good resolution of the dissipative scales is highly desirable, considering the putative exact nature of these relations.

\section{SUMMARY AND CONCLUSIONS}

In terms of new predictions the theory described above has a lot to offer. Firstly, we have presented the fusion rules, and it is extremely worthwhile to test them against experimental data. One can achieve a reasonable test already by using existing data sets from atmospheric boundary layers or grid turbulence. In such experiments one measures usually at one space point as a function of time. Using the standard Taylor hypothesis one can measure many-point correlation functions for points placed along one line. It is possible to examine the properties of such correlation functions when one distance is much smaller than all others. Another prediction pertains to the viscous scaling function and the anomalous exponents $x_{n}$ that characterize them, see Eq.(6.3). To test these predictions one needs a good resolution of the sub-dissipative scales in a high Reynolds number experiment. Such data are not readily available, but very worthwhile to acquire. Another important point raised briefly in this paper has to do with the set of exponents $\beta_{l}$ which govern the anisotropic properties of the correlation functions. We noted elsewhere that the same set of exponents characterizes the correlation functions

\section{ACKNOWLEDGMENTS}

This work was supported in part by the German Israeli Foundation, the US-Israel Bi-National Science Foundation, the Minerva Center for Nonlinear Physics, and the Naftali and Anna Backenroth-Bronicki Fund for Research in Chaos and Complexity.

\section{APPENDIX A: SOME SPECIAL GEOMETRIES OF FUSION AND THEIR IMPLICATIONS}

\section{Special geometry of Fig. A 1}

FIG. A 1. Special geometry of fusion that is discussed in Appendix A 1

As we discussed in subsection II C 2, the evaluation (2.11) is inapplicable when the smallest of the large separations (say $\left|\boldsymbol{r}_{1}-\boldsymbol{r}_{2}\right|=R_{\text {min }}$ ) (which is not associated with a velocity difference) becomes similar to small separations $r$ (across a velocity difference). To understand how to evaluate the correlation function in this case consider the limit $R_{\min }=0$ with the help of the special geometry shown in Fig. A 1 . We have four coordinates, $\boldsymbol{r}_{1}, \boldsymbol{r}_{1}^{\prime}, \boldsymbol{r}_{2}$ and $\boldsymbol{r}_{2}^{\prime}$ organized as shown in the figure, i.e. $\boldsymbol{r}_{1}^{\prime}-\boldsymbol{r}_{1}$ along the $z$-axis, $\boldsymbol{r}_{2}=\boldsymbol{r}_{1}$, and $\boldsymbol{r}_{2}^{\prime}$ is on the $x$-axis, with equal distances to $\boldsymbol{r}_{1}$ and $\boldsymbol{r}_{1}^{\prime}$. This special geometry will help us to derive a result that holds more generally. In addition to the velocity differences $\boldsymbol{w}\left(\boldsymbol{r}_{1}, \boldsymbol{r}_{1}^{\prime}\right)$ and $\boldsymbol{w}\left(\boldsymbol{r}_{2}, \boldsymbol{r}_{2}^{\prime}\right)$ we can have any number of of velocity differences $\boldsymbol{w}\left(\boldsymbol{r}_{i}, \boldsymbol{r}_{i}^{\prime}\right)$, but we demand that the product of all the additional $n-2$ velocity differences (denoted in short-hand as $\{w\}^{n-2}$ ) remains invariant to rotations of $\pi$ radians around the $x$ axis. In Fig. A 1 we show two such additional velocity differences across $\boldsymbol{r}_{3}-\boldsymbol{r}_{3}^{\prime}$ and $\boldsymbol{r}_{4}-\boldsymbol{r}_{4}^{\prime}$. We will write the $n$-th order correlation function in short-hand notation as 


$$
\begin{aligned}
& F_{n}\left(\boldsymbol{r}_{1}, \boldsymbol{r}_{1}^{\prime} ; \boldsymbol{r}_{2}, \boldsymbol{r}_{2}^{\prime} ;\left\{\boldsymbol{r}_{k}, \boldsymbol{r}_{k}^{\prime}\right\}\right) \\
= & \left\langle\boldsymbol{w}\left(\boldsymbol{r}_{1}, \boldsymbol{r}_{1}^{\prime}\right) \cdot \boldsymbol{w}\left(\boldsymbol{r}_{2}, \boldsymbol{r}_{2}^{\prime}\right)\{w\}^{n-2}\right\rangle .
\end{aligned}
$$

By definition $\boldsymbol{w}\left(\boldsymbol{r}_{2}, \boldsymbol{r}_{2}^{\prime}\right)=\boldsymbol{w}\left(\boldsymbol{r}_{2}, \boldsymbol{r}_{1}^{\prime}\right)+\boldsymbol{w}\left(\boldsymbol{r}_{1}^{\prime}, \boldsymbol{r}_{2}^{\prime}\right)$. Remembering that $\boldsymbol{r}_{1}=\boldsymbol{r}_{2}$ we get

$$
\begin{aligned}
& F_{n}\left(\boldsymbol{r}_{1}, \boldsymbol{r}_{1}^{\prime} ; \boldsymbol{r}_{2}, \boldsymbol{r}_{2}^{\prime} ;\left\{\boldsymbol{r}_{k}, \boldsymbol{r}_{k}^{\prime}\right\}\right)=F_{n}\left(\boldsymbol{r}_{1}, \boldsymbol{r}_{1}^{\prime} ; \boldsymbol{r}_{1}^{\prime}, \boldsymbol{r}_{2}^{\prime} ;\left\{\boldsymbol{r}_{k}, \mathbf{r}_{k}^{\prime}\right\}\right) \\
& +F_{n}\left(\boldsymbol{r}_{1}, \boldsymbol{r}_{1}^{\prime} ; \boldsymbol{r}_{1}, \boldsymbol{r}_{1}^{\prime} ;\left\{\boldsymbol{r}_{k}, \boldsymbol{r}_{k}^{\prime}\right\}\right) .
\end{aligned}
$$

Due to the rotation symmetry the first term on the right hand side (RHS) of (A2) may be written as $F_{n}\left(\boldsymbol{r}_{1}, \boldsymbol{r}_{1}^{\prime} ; \mathbf{r}_{1}, \boldsymbol{r}_{2}^{\prime} ;\left\{\boldsymbol{r}_{k}, \boldsymbol{r}_{k}^{\prime}\right\}\right)$, which by definition is the $-F_{n}\left(\boldsymbol{r}_{1}, \boldsymbol{r}_{1}^{\prime} ; \boldsymbol{r}_{2}, \boldsymbol{r}_{2}^{\prime} ;\left\{\boldsymbol{r}_{k}, \mathbf{r}_{k}^{\prime}\right\}\right)$. Finally we derive the identity

$F_{n}\left(\boldsymbol{r}_{1}, \boldsymbol{r}_{1}^{\prime} ; \boldsymbol{r}_{2}, \boldsymbol{r}_{2}^{\prime} ;\left\{\boldsymbol{r}_{k}, \boldsymbol{r}_{k}^{\prime}\right\}\right)=\frac{1}{2} F_{n}\left(\boldsymbol{r}_{1}, \boldsymbol{r}_{1}^{\prime} ; \boldsymbol{r}_{1}, \boldsymbol{r}_{1}^{\prime} ;\left\{\boldsymbol{r}_{k}, \mathbf{r}_{k}^{\prime}\right\}\right)$.

The object on the RHS has two velocity differences across a small separation $r$, and $n-2$ large separations. Therefore it follows the usual fusion rules $(2.8)$ for $p=2$. Accordingly

$$
\begin{aligned}
& \boldsymbol{F}_{n}\left(\boldsymbol{r}_{1}, \boldsymbol{r}_{1}^{\prime} ; \boldsymbol{r}_{2}, \boldsymbol{r}_{2}^{\prime} ;\left\{\boldsymbol{r}_{k}, \mathbf{r}_{k}^{\prime}\right\}\right) \\
= & \left.\tilde{\boldsymbol{F}}_{2}\left(\boldsymbol{r}_{1}, \boldsymbol{r}_{1}^{\prime}\right) \boldsymbol{\Psi}_{n, 2}\left\{\boldsymbol{r}_{k}, \boldsymbol{r}_{k}^{\prime}\right\}\right) \propto\left(\frac{r}{R}\right)^{\zeta_{2}} R^{\zeta_{n}} .
\end{aligned}
$$

This result was derived for the very specific geometry shown in Fig. A 1. However, the last formula holds much more generally. Even if we tilt the vector $\boldsymbol{r}_{1}^{\prime}-\boldsymbol{r}_{1}$ in an arbitrary angle, the result remains invariant. The reason is that in the limit $r \ll R$ the anisotropic effects of the large scales on the small scales have already disappeared, as is shown in Section III. If we ruin the symmetry of rotation around the $x$-axis we also do not change the final result. The change will be in the factor in Eq. A3 from $1 / 2$ to a geometry-dependent factor of the order of unity. We can even dissociate $\boldsymbol{r}_{2}$ from $\boldsymbol{r}_{1}$ over distances of the order of $\left|\boldsymbol{r}_{1}^{\prime}-\boldsymbol{r}_{1}\right|$. Eq. A4 is rather universal.

\section{Special geometry of Fig. A 2}

FIG. A 2. Special geometry of fusion that is discussed in Appendix A 2

In Fig. A 2 we show a situation in which there exist two velocity differences across short distances. The difference with the general situation shown in Fig. 1 (for $p=2$ ) is that we have a fusion of points belonging to short and long separations, i.e $\boldsymbol{r}_{2}=\boldsymbol{r}_{3}$. The same type of fusion existed also in Fig. A 1, but there was only one one short distance with a velocity difference across it. Now we have two. According to the general rule we should have a contribution that is proportional to $r^{\zeta_{2}}$. The aim of this special discussion is to show that in this case there exists a subleading contribution that is proportional to $r^{\zeta_{3}}$. This is important in the analysis that involves the calculation of gradients with respect to these positions. To this aim consider the correlation function

$$
\begin{aligned}
& F_{n}\left(\boldsymbol{r}_{1}, \boldsymbol{r}_{1}^{\prime} ; \boldsymbol{r}_{2}, \boldsymbol{r}_{2}^{\prime} ; \boldsymbol{r}_{3}, \boldsymbol{r}_{3}^{\prime} ;\left\{\boldsymbol{r}_{k}, \boldsymbol{r}_{k}^{\prime}\right\}\right) \\
= & \left\langle\left[\boldsymbol{w}\left(\boldsymbol{r}_{1}, \boldsymbol{r}_{1}^{\prime}\right) \cdot \boldsymbol{w}\left(\boldsymbol{r}_{2}, \boldsymbol{r}_{2}^{\prime}\right)\right]\left[\boldsymbol{w}\left(\boldsymbol{r}_{3}, \boldsymbol{r}_{3}^{\prime}\right) \cdot \hat{\boldsymbol{z}}\right]\{w\}^{n-3}\right\rangle
\end{aligned}
$$

where $\hat{\boldsymbol{z}}$ is a unit vector in the $z$ direction. We again use a short hand notation $\{w\}^{n-3}$ for the product of $n-3$ velocity differences across large separations which depend on the coordinates $\boldsymbol{r}_{4}, \boldsymbol{r}_{4}^{\prime}$ and higher. Using now the fact that $\boldsymbol{w}\left(\boldsymbol{r}_{3}, \boldsymbol{r}_{3}^{\prime}\right)=\boldsymbol{w}\left(\boldsymbol{r}_{3}, \boldsymbol{r}_{1}^{\prime}\right)+\boldsymbol{w}\left(\boldsymbol{r}_{1}^{\prime}, \boldsymbol{r}_{3}^{\prime}\right)$ we compute

$$
\begin{aligned}
& F_{n}\left(\boldsymbol{r}_{1}, \boldsymbol{r}_{1}^{\prime} ; \boldsymbol{r}_{2}, \boldsymbol{r}_{2}^{\prime} ; \boldsymbol{r}_{3}, \boldsymbol{r}_{3}^{\prime} ;\left\{\boldsymbol{r}_{k}, \boldsymbol{r}_{k}^{\prime}\right\}\right) \\
= & \left\langle\left[\boldsymbol{w}\left(\boldsymbol{r}_{1}, \boldsymbol{r}_{1}^{\prime}\right) \cdot \boldsymbol{w}\left(\boldsymbol{r}_{2}, \boldsymbol{r}_{2}^{\prime}\right)\right]\left[\boldsymbol{w}\left(\boldsymbol{r}_{1}^{\prime}, \boldsymbol{r}_{3}^{\prime}\right) \cdot \hat{\boldsymbol{z}}\right]\{w\}^{n-3}\right\rangle \\
& +\left\langle\left[\boldsymbol{w}\left(\boldsymbol{r}_{1}, \boldsymbol{r}_{1}^{\prime}\right) \cdot \boldsymbol{w}\left(\boldsymbol{r}_{2}, \boldsymbol{r}_{2}^{\prime}\right)\right]\left[\boldsymbol{w}\left(\boldsymbol{r}_{3}, \boldsymbol{r}_{1}^{\prime}\right) \cdot \hat{\boldsymbol{z}}\right]\{w\}^{n-3}\right\rangle .
\end{aligned}
$$

Rotating around the $x$-axis we can rewrite the last correlator on the RHS of (A6) as the correlator on the LHS with an opposite sign. To see this note that all the terms are invariant except the term $\boldsymbol{w}\left(\boldsymbol{r}_{3}, \boldsymbol{r}_{1}^{\prime}\right) \cdot \hat{\mathbf{z}}$ that changes sign. Thus finally

$$
\begin{aligned}
& F_{n}\left(\boldsymbol{r}_{1}, \boldsymbol{r}_{1}^{\prime} ; \boldsymbol{r}_{2}, \boldsymbol{r}_{2}^{\prime} ; \boldsymbol{r}_{3}, \boldsymbol{r}_{3}^{\prime} ;\left\{\boldsymbol{r}_{k}, \boldsymbol{r}_{k}^{\prime}\right\}\right) \\
= & \frac{1}{2}\left\langle\left[\boldsymbol{w}\left(\boldsymbol{r}_{1}, \boldsymbol{r}_{1}^{\prime}\right) \cdot \boldsymbol{w}\left(\boldsymbol{r}_{2}, \boldsymbol{r}_{2}^{\prime}\right)\right]\left[\boldsymbol{w}\left(\boldsymbol{r}_{1}^{\prime}, \boldsymbol{r}_{3}^{\prime}\right) \cdot \hat{\boldsymbol{z}}\right]\{w\}^{n-3}\right\rangle
\end{aligned}
$$

This correlator has three explicit velocity differences across short distances, and therefore according to the general rule with $p=3$ it is proportional to $r^{\zeta_{3}}$. In the general case without rotation symmetry the leading term $r^{\zeta_{2}}$ remains. Therefore we conclude that in the geometry in which there are two velocity differences across a small separation and one point belonging to a velocity difference across a large separation (see Fig. A 2), the correlation function $F_{n}$ can be written to leading order as

$$
\begin{aligned}
& \boldsymbol{F}_{n}\left(\boldsymbol{r}_{1}, \boldsymbol{r}_{1}^{\prime} ; \boldsymbol{r}_{2}, \boldsymbol{r}_{2}^{\prime} ; \boldsymbol{r}_{3}, \boldsymbol{r}_{3}^{\prime} ;\left\{\boldsymbol{r}_{k}, \boldsymbol{r}_{k}^{\prime}\right\}\right)=\left[\tilde{\boldsymbol{S}}_{2}\left(\left|\boldsymbol{r}_{1}-\boldsymbol{r}_{2}\right|\right)\right. \\
& \left.+\tilde{\boldsymbol{S}}_{2}\left(\left|\boldsymbol{r}_{1}^{\prime}-\boldsymbol{r}_{2}^{\prime}\right|\right)-\tilde{\boldsymbol{S}}_{2}\left(\left|\boldsymbol{r}_{1}-\boldsymbol{r}_{2}^{\prime}\right|\right)-\tilde{\boldsymbol{S}}_{2}\left(\left|\boldsymbol{r}_{1}^{\prime}-\boldsymbol{r}_{2}\right|\right)\right] \\
& \boldsymbol{\Psi}_{n, 2}\left(\boldsymbol{r}_{0}, \boldsymbol{r}_{3}^{\prime} ;\left\{\boldsymbol{r}_{k}, \boldsymbol{r}_{k}^{\prime}\right\}\right)+\left[\tilde{\boldsymbol{S}}_{3}\left(\boldsymbol{r}_{0} \mid \boldsymbol{r}_{1}, \boldsymbol{r}_{2}, \boldsymbol{r}_{3}\right)+\tilde{\boldsymbol{S}}_{3}\left(\boldsymbol{r}_{0} \mid \boldsymbol{r}_{1}^{\prime}, \boldsymbol{r}_{2}^{\prime}, \boldsymbol{r}_{3}\right)\right. \\
& \left.-\tilde{\boldsymbol{S}}_{3}\left(\boldsymbol{r}_{0} \mid \boldsymbol{r}_{1}^{\prime}, \boldsymbol{r}_{2}, \boldsymbol{r}_{3}\right)-\tilde{\boldsymbol{S}}_{3}\left(\boldsymbol{r}_{0} \mid \boldsymbol{r}_{1}, \boldsymbol{r}_{2}^{\prime}, \boldsymbol{r}_{3}\right)\right] \boldsymbol{\Psi}_{n, 3}\left(\boldsymbol{r}_{0},\left\{\boldsymbol{r}_{k}, \boldsymbol{r}_{k}^{\prime}\right\}\right),
\end{aligned}
$$

where $\boldsymbol{r}_{0}=\left[\boldsymbol{r}_{1}+\boldsymbol{r}_{1}^{\prime}+\boldsymbol{r}_{2}+\boldsymbol{r}_{2}^{\prime}+\boldsymbol{r}_{3}\right] / 5$, and $\tilde{\boldsymbol{S}}_{2}, \tilde{\boldsymbol{S}}_{3}, \boldsymbol{\Psi}_{n, 2}$ and $\boldsymbol{\Psi}_{n, 3}$ are homogeneous functions of their arguments (in the inertial interval) with scaling exponents $\zeta_{2}, \zeta_{3}$, $\zeta_{n}-\zeta_{2}$ and $\zeta_{n}-\zeta_{3}$ respectively. The function $\tilde{\boldsymbol{S}}_{3}$ may be different from the function $S_{3}$ in its dependence on the angles and the ratios between its argument coordinates. But they share the same scaling exponent.

\section{Special geometry of Fig. A 3}


FIG A3. Special geometry of fusion that is discussed in Appendix A 3

In this subsection we consider the case in which there are four coordinates within the ball of size $r$, but only two coordinates $\left(\boldsymbol{r}_{1}\right.$ and $\left.\boldsymbol{r}_{1}^{\prime}\right)$ belong to a velocity difference, see Fig. IIE. The other two coordinates $\left(\boldsymbol{r}_{2}\right.$ and $\left.\boldsymbol{r}_{3}\right)$ are in the ball, but they relate to velocity differences across large separations. To understand the situation we again consider a special geometry, that of Fig. A 3. In this geometry $\boldsymbol{r}_{1}=\boldsymbol{r}_{2}=\boldsymbol{r}_{3}$ and we study the correlation function

$$
\begin{aligned}
& \boldsymbol{F}_{n}\left(\boldsymbol{r}_{1}, \boldsymbol{r}_{1}^{\prime} ; \boldsymbol{r}_{2}, \boldsymbol{r}_{2}^{\prime} ; \boldsymbol{r}_{3}, \boldsymbol{r}_{3}^{\prime} ;\left\{\boldsymbol{r}_{k}, \boldsymbol{r}_{k}^{\prime}\right\}\right)=\left\langle\left[\boldsymbol{w}\left(\boldsymbol{r}_{1}, \boldsymbol{r}_{1}^{\prime}\right) \cdot \hat{\boldsymbol{z}}\right]\right. \\
& \left.\left[\boldsymbol{w}\left(\boldsymbol{r}_{2}, \boldsymbol{r}_{2}^{\prime}\right) \cdot \boldsymbol{w}\left(\boldsymbol{r}_{3}, \boldsymbol{r}_{3}^{\prime}\right)\right]\{w\}^{n-3}\right\rangle .
\end{aligned}
$$

Making the substitutions

$$
\begin{aligned}
& \boldsymbol{w}\left(\boldsymbol{r}_{2}, \boldsymbol{r}_{2}^{\prime}\right)=\boldsymbol{w}\left(\boldsymbol{r}_{1}, \boldsymbol{r}_{2}^{\prime}\right)=\boldsymbol{w}\left(\boldsymbol{r}_{1}, \boldsymbol{r}_{1}^{\prime}\right)+\boldsymbol{w}\left(\boldsymbol{r}_{1}^{\prime}, \boldsymbol{r}_{2}^{\prime}\right) \\
& \boldsymbol{w}\left(\boldsymbol{r}_{3}, \boldsymbol{r}_{3}^{\prime}\right)=\boldsymbol{w}\left(\boldsymbol{r}_{1}, \boldsymbol{r}_{3}^{\prime}\right)=\boldsymbol{w}\left(\boldsymbol{r}_{1}, \boldsymbol{r}_{1}^{\prime}\right)+\boldsymbol{w}\left(\boldsymbol{r}_{1}^{\prime}, \boldsymbol{r}_{3}^{\prime}\right)
\end{aligned}
$$

we find that

$$
\begin{aligned}
& 2 \boldsymbol{F}_{n}\left(\boldsymbol{r}_{1}, \boldsymbol{r}_{1}^{\prime} ; \boldsymbol{r}_{2}, \boldsymbol{r}_{2}^{\prime} ; \boldsymbol{r}_{3}, \boldsymbol{r}_{3}^{\prime} ;\left\{\boldsymbol{r}_{k}, \boldsymbol{r}_{k}^{\prime}\right\}\right)=\left\langle\left[\boldsymbol{w}\left(\boldsymbol{r}_{1}, \boldsymbol{r}_{1}^{\prime}\right) \cdot \hat{\boldsymbol{z}}\right]\right. \\
& \left.\times\left|\boldsymbol{w}\left(\boldsymbol{r}_{1}, \boldsymbol{r}_{1}^{\prime}\right)\right|^{2}\{w\}^{n-3}\right\rangle+\left\langle\left[\boldsymbol{w}\left(\boldsymbol{r}_{1}, \boldsymbol{r}_{1}^{\prime}\right) \cdot \hat{\boldsymbol{z}}\right]\right. \\
& \left.\times \boldsymbol{w}\left(\boldsymbol{r}_{1}, \boldsymbol{r}_{1}^{\prime}\right) \cdot\left[\boldsymbol{w}\left(\boldsymbol{r}_{1}^{\prime}, \boldsymbol{r}_{2}^{\prime}\right)+\boldsymbol{w}\left(\boldsymbol{r}_{1}^{\prime}, \boldsymbol{r}_{3}^{\prime}\right)\right]\{w\}^{n-3}\right\rangle . \quad(\mathrm{A} 12)
\end{aligned}
$$

In obtaining this equation we used the symmetry under rotation around the $x$ axis in $\pi$. Under this rotation $\boldsymbol{r}_{1} \rightarrow \boldsymbol{r}_{1}^{\prime}$. The first term on the RHS has an explicit product of three velocity differences across a small distance, and it is therefore proportional to $r^{\zeta_{3}}$. The second term has two velocity differences across a small scale, and according to the previous subsection it contains two contributions, one proportional to $r^{\zeta_{2}}$ and the other to $r^{\zeta_{3}}$. One can write it in a form similar to (39) and (40), but this is not needed at the moment.

[1] V.S. L'vov and I. Procaccia "Exact Resummation in the Theory of Hydrodynamic Turbulence": 0. Line Resummed Diagrammatic Perturbation Approach" in F. David P. Ginsparg, and J. Zinn-Justin, eds. Les Houches session LXII, 1994, "Fluctuating Geometries in Statistical Mechanics and Field Theory" (Elsevier, 1995).
[2] V.S. L'vov and I. Procaccia, "Exact Resummation in the Theory of Hydrodynamic Turbulence": I. The Ball of Locality and Normal Scaling",Phys. Rev. E, 52, 3840 (1995).

[3] V.S. L'vov and I. Procaccia, "Exact Resummation in the Theory of Hydrodynamic Turbulence": II. The Ladder to Anomalous Scaling",Phys. Rev. E, 52, 3858 (1995).

[4] V.S. L'vov and I. Procaccia, "Exact Resummation in the Theory of Hydrodynamic Turbulence": III.Scenarios for Multiscaling and Intermittency". Phys. Rev. E, 53,3468 (1996).

[5] V.S. L'vov and I. Procaccia, "Fusion Rules in Turbulent Systems with Flux Equilibrium", Phys. Rev. Lett. 1996, 76, 2896 (1996).

[6] V.S. L'vov and I. Procaccia, "The universal scaling exponents of anisotropy and their measurement", Phys. Fluids, in press (1996).

[7] V.S. L'vov, E. Podivilov and I. Procaccia, "Scaling behavior in turbulence is doubly anomalous", Phys. Rev. Lett., 76, 3963 (1996). .

[8] V.S. L'vov and I. Procaccia, "The viscous scales in hydrodynamic turbulence are anomalous scaling functions", Phys. Rev. Lett., submitted.

[9] A. S. Monin and A. M. Yaglom. Statistical Fluid Mechanics: Mechanics of Turbulence, volume II. (MIT Press, Cambridge, Mass., 1973).

[10] F. Anselmet, Y. Gagne, E.J. Hopfinger, and R.A. Antonia, J. Fluid Mech. 140, 63 (1984).

[11] E.A. Kuznetsov and V.S. L'vov, Physica 2D 203 (1981).

[12] K.R. Sreenivasan and P. Kailasnath. Phys. Fluids A 5, 512, (1993).

[13] R. Benzi, S. Ciliberto, R. Tripiccione, C. Baudet, F. Massaioli and S. Succi, Phys. Rev. E 48, R29 (1993).

[14] Uriel Frisch. Turbulence: The Legacy of A.N. Kolmogorov. (Cambridge University Press, Cambridge, 1995).

[15] A.N. Kolmogorov, Dokl. Acad. Nauk SSSR, 30, 9 (1941).

[16] V.S. L'vov and V. Lebedev, Europhys. Lett. 29, 681 (1995).

[17] A.A.Praksovsly, Phys. Fluids A4, 2589 (1992)

[18] A. L. Fairhall, O. Gat, V.S. L'vov and I. Procaccia, Phys. Rev. E 533518 (1996).

[19] R.H. Kraichnan, Phys. Fluids, 8, 575 (1965).

[20] S. Grossmann, D. Lohse, V.S. L'vov and I. Procaccia, Phys. Rev. Lett. 73, 432 (1994)

[21] G. Falkovich and V.S. L'vov, Chaos, Solitons and Fractals, 5, 1855 (1995).

[22] G. Paladin and A. Vulpiani, Phys. Rev. A35, 1971 (1987).

[23] U. Frisch and M. Vergassola, Europhys. Lett. 14, 439 (1991). 